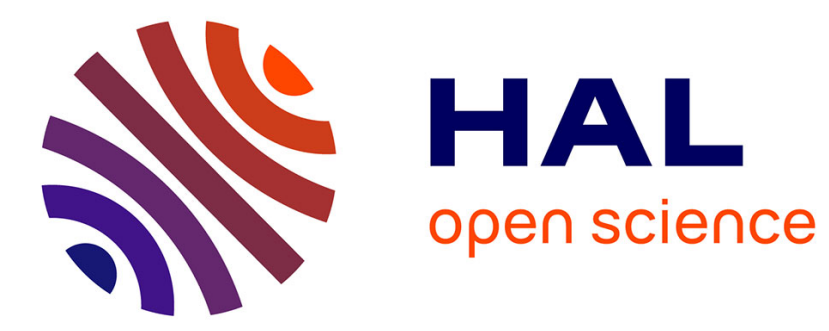

\title{
POD-Spectral Decomposition for Fluid Flow Analysis and Model Reduction
}

Ada Cammilleri, Florimond Guéniat, Johan Carlier, Luc R. Pastur, Etienne Mémin, François Lusseyran, Guillermo Artana

\section{To cite this version:}

Ada Cammilleri, Florimond Guéniat, Johan Carlier, Luc R. Pastur, Etienne Mémin, et al.. PODSpectral Decomposition for Fluid Flow Analysis and Model Reduction. Theoretical and Computational Fluid Dynamics, 2013, 27 (125), pp.787-815. 10.1007/s00162-013-0293-2 . hal-00793380

\section{HAL Id: hal-00793380 \\ https://hal.inria.fr/hal-00793380}

Submitted on 22 Feb 2013

HAL is a multi-disciplinary open access archive for the deposit and dissemination of scientific research documents, whether they are published or not. The documents may come from teaching and research institutions in France or abroad, or from public or private research centers.
L'archive ouverte pluridisciplinaire HAL, est destinée au dépôt et à la diffusion de documents scientifiques de niveau recherche, publiés ou non, émanant des établissements d'enseignement et de recherche français ou étrangers, des laboratoires publics ou privés. 
A. Cammilleri - F. Gueniat • J. Carlier • L. Pastur .

E. Memin - F. Lusseyran - G. Artana

\title{
POD-Spectral Decomposition for Fluid Flow Analysis and Model Reduction
}

Received: date / Accepted: date

\begin{abstract}
We propose an algorithm that combines Proper Orthogonal Decomposition with a spectral method to analyse and extract from time data series of velocity fields, reduced order models of flows. The flows considered in this study are assumed to be driven by non linear dynamical systems exhibiting a complex behavior within quasi-periodic orbits in the phase space. The technique is appropiate to achieve efficient reduced order models even in complex cases for which the flow description requires a discretization with a fine spatial and temporal resolution. The proposed analysis enables to decompose complex flow dynamics into modes oscillating at a single frequency. These modes are associated with different energy levels and spatial structures. The approach is illustrated using time resolved PIV data of a cylinder wake flow with associated Reynolds number equal to 3900.
\end{abstract}

Keywords Reduced Order Modeling · POD · DMD · Spectral Analysis

PACS 47.27-ed $\cdot$ 47.27.De $\cdot$ 47.85.L-

\section{Introduction}

The reduced order modeling of fluid flow dynamics is of major interest in order to supply computationally affordable dynamical systems that may be used within data assimilation procedures [29, 7] or flow control strategies [18] for forecasting new or intermediate states of the flow. Such models generally rely on the use of a sequence of velocity fields to build a low dimensional empirical basis of the flow dynamics. Those velocity fields can be either supplied from numerical simulations or from physical experiments through velocimetry measurement techniques, although in the latter case noise comes into the picture and makes the problem much harder. The basis functions associated with such reduced modeling provide in addition a compact kinematical description of the flow that can be used for analysis or inspection purposes. In this latter case, one is interested in recovering or characterizing specific dynamical instabilities or coherent structures to yield an insight in terms of distinct physical events involved within the flow of interest.

This research has been funded by argentine and french governments through grants LIA FMF/PMF, Huracan, PIP 3303 and UBACYT IN017.

A. Cammilleri and G. Artana

LFD-F.I.UNIVERSIDAD DE BUENOS AIRES-CONICET-ARGENTINA

Tel.: +54-11-43430092

Fax: +54-11-43311852

E-mail: gartana@fi.uba.ar

F. Gueniat, L. Pastur and F. Lusseyran

UNIVERSITY PARIS SUD 11- LIMSI-CNRS-FRANCE

J. Carlier and E. Memin

FLUMINANCE-INRIA-RENNES- FRANCE 
Different approaches have been proposed so far for the constitution of low order empirical dynamical models of fluid flows from time series data. Among them, one of the most popular approach has been to consider a Karhunen-Loeve decomposition of the data series [19]. This strategy, referred to as Proper Orthogonal Decomposition (POD), supplies a compact flow description in term of topos (modes of the spatial basis), that are ranked by their associated fluctuating kinetic energy. The most energetic modes correspond generally to large scale structures but are generally not representative of distinct physical events in the frequency domain. It is hence sometimes difficult to associate the set of POD modes with clear distinguishable flow dynamical features or events. The correspondence of these modes with the so called coherent structures of the flow reveals thus sometimes questionable. Only in the special case of homogeneous correlation tensor (applicable for instance in isotropic turbulence, or flows with a preferential direction of homogeneity [20] or with translational symmetries [26]) the POD basis reduces to the Fourier modes [10]. However, in that case one loses spatial localization, which characterizes the coherent structures of interest.

Reduced order dynamics is formally obtained after a Galerkin projection of the Navier Stokes equations in the subspace spanned by the POD modes. This approach turns the partial differential equations (PDE) description of the flow dynamics into a coupled system of ordinary differential equations (ODE) involving the POD temporal coefficients - called chronos. The dimension of the system depends on the number of components retained for the analysis. This degree of freedom is usually determined from an energetic criterion. Obviously, those models are strictly dedicated to a given flow situation. This introduces an important drawback for flow control applications and has motivated different strategies to improve the expansion basis like balanced proper orthogonal decomposition [6] or frequential snapshots [11].

A potential pitfall of POD-ROM technique lies in the numerical stability of the system extracted. One of the most important causes for this loss of stability is related to truncation of low energy modes, which as a result suppresses damping associated with energetic dissipation mechanism. It is thus tempting, on the one hand, to keep a great number of modes (that is at most given by the number of snapshots). On the another hand, the requirement of reducing the system and the difficulty to accurately extract low energetic modes (associated with weak eigenvalues of the data autocorrelation matrix) encourages to discard the lowest energetic modes. In worst cases, e.g. when the dimension of the model becomes too large, the model predictions of the flow dynamics are prone to blow up even at very short time horizons.

Different variations of the POD-Galerkin procedure have been proposed to overcome these limitations. Some of them include additional dissipative models [12] or rely on nonlinear Galerkin projection techniques $[1,4,13]$. Robust techniques based on optimal control strategies have been also proposed for building reduced dynamical models from noisy data $[9,15]$ and incomplete knowledge of the actual flow dynamics (i.e. unknown initial condition, unknown or partially known forcing terms,...). Those techniques have demonstrated to be able to extract very accurately low order dynamical system on the time range on which the data are available but may experience some limitations for forecasting new states of the system. Techniques to improve the stability of the reduced order model (ROM) have been recently proposed for wake flows $[4,27]$. This is the kind of flow we consider in this work to illustrate our approach even if the technique we propose could apply to any flow exhibiting a periodic character in the phase space.

In this contribution, we aim at identifying a ROM whose dynamics is not obtained by integrating in time a system of differential equations, but rather by performing matrix multiplication. At the same time, we look for a model whose reduction relies on physical criterion, combining both energy considerations and dynamical relevance. This is achieved by designing a model that relies on both POD and dynamic mode decomposition (DMD).

DMD has been recently introduced in the field of fluid mechanics by Schmid [24] and Rowley [5] to identify modes relevant with respect to the hidden system dynamics. The basic idea consists to postulate the existence of an evolution operator that transforms the current observable snapshot most of the time a velocity snapshot - into the next one. This imposes the operator to be stationary, i.e. constant in time. As a consequence, snapshots corresponding to an underlying non-linear dynamics, are vectors lying in a vector space in which the evolution operator enables to map one vector into another. DMD has been successfully applied to various physical configurations in order to identify coherent structures in flows that have associated a well-defined temporal frequency [6,23,22]. 
The DMD procedure assumes that for the phenomenon under study the $N^{t h}$ snapshot is a linear combination of the first $(N-1)$ ones. This is equivalent to consider that the $N-1$ first snapshots form a basis on which any new snapshot can be decomposed. Such an hypothesis is hardly satisfied, and as a consequence in general the decomposition generates a residual. The dimension of the residual space depends on the difference between the observable dimension and the number of snapshots. The greater this difference the larger the residual dimension. The residual however will be assumed to be almost negligible and thus not taken into account in the analysis.

The determination of the dynamic modes of this analysis requires estimating the (complex) eigenvalues of the evolution operator. The modes that are obtained with a DMD oscillate with a single frequency that is determined by the eigenvalue associated with the mode. The eigenvalues, whose number depends on the operator dimension, cannot be determined directly from the evolution operator as this operator is in general unknown. It is however possible to propose techniques that enables to estimate some of these eigenvalues with matrix that are similar to the evolution operator (the number of approximated eigenvalues agrees with the number of considered snapshots). In the usual case, when the number of snapshots is lower than the dimension of the evolution operator, only some of the total number of eigenvalues can be captured. As a result, in this situation any spectrum built considering all the approximated eigenvalues may be incomplete as some frequency channels will be missing.

When the flow regime is perfectly permanent, theoretically the eigenvalues should map on the unit circle of the complex plane. In this situation it is possible to define a power spectrum by associating the $\mathcal{L}_{2}$-norm of any given dynamic mode to the frequency channel associated with the eigenvalue. In a periodic regime case and when the data set under consideration is centered (vanishing average), Chen [17] indicates that discrete Fourier transform (DFT) and DMD constitute formally equivalent methods. We can mention however that this equivalence only applies when the number of snapshots is lower than the dimension of the snapshot vector.

In most practical situations, eigenvalues are not strictly unitary and even when they lie in the vicinity of the unit circle, it addresses the question of the meaning of the $\mathcal{L}_{2}$-norm. For instance, the contribution to the dynamics of a mode whose eigenvalue is smaller than 1 , may be significant on short time range, while it is rapidly negligible over a larger time horizon. Hence, identification of power spectrum with the mode amplitude spectrum may become questionable in such situations. One way to recover a spectrum similar to a power spectrum would be to balance the $\mathcal{L}_{2}$-norm of the mode such as to consider its mean contribution over the observation time range. Unfortunately, straightforward calculus can show that no simple balance procedure can be derived.

In this study, we propose to build reduced order models that rely on the dynamic mode decomposition of datasets of the POD chronos. The evolution operator associated with this procedure enables to express any new set of chronos in terms of the preceding ones. This change of point of view of the DMD has some important consequences. The evolution operator is no longer acting on a restriction of the Identity operator on the experimental space - the velocity field - but on others observables: the chronos. With POD we get an orthogonal basis that ranks modes according to an energy criterion whereas DMD enables identifying, among chronos, those that are relevant with respect to their spectral contribution. Hence, a direct link to the kinetic energy spectra can be established. In the following, the technique will be referred to as Chronos-Koopman Spectral (CKS) analysis.

In the same way as for DMD, the approach we propose in this work decomposes the flow dynamics into modes that oscillate at a single frequency, however their energy levels and their relative contribution to the total flow energy can be clearly identified enabling to construct suitable power spectra. All the attractive properties in terms of instability analysis of DMD analysis are naturally kept by the CKS decomposition. The spatial structure of the dominant monofrequential modes can still be easily distinguished and extracted.

To minimize the residual vector and to approximate the larger number of eigenvalues, DMD technique requires that the number of snapshots to be of the same order than the dimension of snapshot vector. The method we propose does not present this strong constraint. The technique enables to approximate almost the same number of eigenvalues of the operator it considers and the residuals generated by the use of this approach will always be restricted to lie in a unidimensional space. In cases in which the number of snapshots is much lower than the spatial dimension of the snapshots the CKS will allow us coping with a simpler and better posed problem than traditional DMD. Similar conditions can be attained for both approaches only by enlarging the dataset. In general, when this will be possible it will be however paid by a higher computational cost. 
As we will show with CKS technique, predicting the chronos values does not require any timeintegration but simple matrix multiplications. Moreover because eigenvalues lie in proximity of the unit circle, the model have associated modes with very low growth (or decay) rates. Hence, the dynamics does not blow up in general over time scales several times larger than the data set time range. This decomposition provides in a natural way a reduced order model of the chronos dynamics over long time range without any need of tuning of stabilizing parameters.

The remainder of the manuscript is organized as follows. In section 2 we briefly recall the principles governing the Proper Orthogonal Decomposition and the Dynamic Mode Decomposition. Section 3 focuses on the Chronos- Koopman Spectral analysis, that will be applied, in section 4, to the model reduction of a wake behind a cylinder. In particular, we discuss the physical meaning of the $\mathcal{L}_{2^{-}}$ norm of dynamic modes with respect to the energy content of the data set. We show that it is more appropriate to perform DMD on the POD time-coefficients when one aims at devising a reduced order model combining both an energy criterion and spectral relevance.

The flow considered in section 4 corresponds to a three dimensional flow in which the vortex shedding imposes a fundamental dominant frequency accompanied with frequencies associated with instabilities of the shear layer. CKS analysis is done on an experimental dataset made of time-resolved velocity fields. We will see that the basic features of the energy frequency spectrum of the wake flow, at the Reynolds number under consideration, are effectively well recovered with the CKS analysis whereas it is not the case when applying the standard procedure of DMD. Section 5 is dedicated to a summary of our results and opens further discussions.

\section{Modal Decomposition Methods}

In this section a brief introduction is given about the Proper Orthogonal Decomposition and the spectral method based on Koopman operator theory. Thorough descriptions of the latter decomposition can be found in $[6,22,23]$. Readers interested on theoretical aspects of the Koopman operator may also refer to $[14,3]$.

\subsection{Proper Orthogonal Decomposition}

The POD-Galerkin method has been extensively described in many articles. The method foundations are for instance thoroughly described in [19]. Let us review very briefly its main characteristics when it is computed from a sequence of 2 components of velocity fields contained in a slice of the flow. Extension to 3 components and multi-slice cases is immediate.

Considering $\Omega \in \mathbb{R}^{2}$ a physical domain with boundary $\partial \Omega$, we assume that we have at our disposal the following time series of measured velocity flow field $\underline{u}(\underline{x}, t) \in L^{2}(\Omega)$

$\mathcal{H}=\left\{\underline{u}\left(\underline{x}, t_{0}\right) ; \underline{u}\left(\underline{x}, t_{1}\right) ; \underline{u}\left(\underline{x}, t_{2}\right) \ldots ; \underline{u}\left(\underline{x}, t_{N}\right)\right\}=\left\{\underline{u}^{0}(\underline{x}) ; \underline{u}^{1}(\underline{x}) ; \underline{u}^{2}(\underline{x}) ; \ldots ; \underline{u}^{N}(\underline{x})\right\}$

where $N+1$ is the total number of snapshots that are assumed to be regularly spaced in time. We look for a decomposition of the flow on a finite set of a $N+1$ orthonormal spatial basis functions

$\left\{\underline{\Phi}_{i}(\underline{x}): \Omega \subset \mathbb{R}^{2} \rightarrow \mathbb{R}^{2}, i=1, \ldots, N+1\right\}$

with temporal coefficients

$\left\{a_{i}(t): \mathbb{R}^{+} \rightarrow \mathbb{R}, i=1, \ldots, N+1\right\}$

such that

$\underline{u}(\underline{x}, t)=\langle\underline{u}(\underline{x})\rangle+\sum_{i=1}^{N+1} a_{i}(t) \underline{\Phi}_{i}(\underline{x})$,

where

$\langle\underline{u}(\underline{x})\rangle=\lim _{T \rightarrow \infty} \frac{1}{T} \int \underline{u}(\underline{x}, t) d t$. 
The POD basis is constructed with the objective of minimizing the projection error

$\epsilon^{2}=\left\langle\int_{\Omega}\left|\underline{u}(\underline{x}, t)-\langle\underline{u}(\underline{x})\rangle-\sum_{i=1}^{N+1} a_{i}(t) \underline{\Phi}_{i}(\underline{x})\right|^{2} d \underline{x}\right\rangle$,

Let us note $D=\left(d_{k j}\right)$ the $(N+1) \times(N+1)$ symmetric and semi-definite positive two-time correlation matrix defined as

$d_{k j}=\frac{1}{N+1} \int_{\Omega} \underline{u}^{\prime k}(\underline{x}) \underline{u}^{\prime j}(\underline{x}) d \underline{x}$,

where we denoted $\underline{u}^{\prime i}(\underline{x})=\underline{u}^{i}(\underline{x})-\langle\underline{u}(\underline{x})\rangle$.

It is well known that the solution of the optimization problem is provided by the $N+1$ eigenfunctions $a_{1}(),. a_{2}(),. \ldots, a_{N+1}($.$) associated with the \varsigma_{1} \geq \varsigma_{2} \geq \ldots \varsigma_{N+1}$ singular values of $D$. This method, known in the literature as the snapshot methods [26], is well suited to vector fields sequences defined on a time range much lower than the velocity field spatial dimension.

The POD modes of the velocity can then be expressed as

$\underline{\Phi}_{i}(\underline{x})=\frac{1}{(N+1)} \sum_{k=0}^{N} \frac{1}{\varsigma_{i}} a_{i}\left(t_{k}\right) \underline{u}^{\prime k}(\underline{x}) \quad$ for $\mathrm{i}=1, \ldots, N+1$.

The Galerkin projection of the Navier-Stokes equations, considering the representation (4), onto the subspace spanned by the reduced set of orthonormal POD modes $\left\{\underline{\Phi}_{1}(\underline{x}), \ldots, \underline{\Phi}_{M}(\underline{x})\right\}$ with $M \leq N+1$ results in a system of coupled ordinary differential equations (ODE) describing the temporal evolution of coefficients $a_{i}(t)$ associated with the $M$ modes retained in the POD basis. In a compact form this system reads

$\frac{d \underline{a}}{d t}=\underline{\dot{a}}=\mathbb{F}(\underline{a})$,

where $\mathbb{F}$ represents a nonlinear operator that can be expressed as a polynomial in $\underline{a}=\left(a_{1} \ldots a_{M}\right)^{T}$. The resulting reduced model generally matches only roughly the flow dynamics as contributions of the discarded modes are removed from this description. Obviously, one seek to remove the modes with the weakest contribution on the dynamics. As the only truncature criterion at hand is an energetic criterion, usually a remotion of POD modes with low eigenvalues is done. However, these modes may correspond to small scales flow events, and their effect on dynamics may be quite important if they are related for instance to energy dissipation mechanisms. As a consequence the temporal integration of the reduced system derived from Galerkin projection may reveal to be unstable and prevents in practice any direct integration over a long time intervals.

\subsection{Spectral mode decomposition based on Koopman operator}

This second family of techniques relies on a spectral decomposition inspired on the Koopman operator theory. This operator is the adjoint of the Frobenius-Perron operator, also called transfer operator, which corresponds to the push-forward measure with respect to a non singular measurable transformation. The Koopman operator describes the evolution of an observer function under the action of the same transformation. These unitary operators are intensively used to study the asymptotic behavior of chaotic deterministic system from a statistical point of view $[16,14]$.

As a matter of example, let us consider a $3 \mathrm{D}$ flow in a $3 \mathrm{D}$ domain discretized on a grid of $n_{g}$ points. The flow dynamics determined by the Navier Stokes equation in the corresponding continuous domain of interest can then be represented by a system of ordinary differential equations for each point of the grid. It is then possible to associate to the flow, a dynamical system in which a given state at time $t$ is defined by the state variable $\underline{X} \in \mathbb{R}^{n}$ such that

$\frac{d \underline{X}}{d t}=h(\underline{X})$. 
For the state space $\mathbb{R}^{n}$ is considered the usual inner product and its norm.

The evolution of the state variable at discrete equidistant points in time, can be then expressed as the mapping,

$\underline{X}^{k+1}=f\left(\underline{X}^{k}\right) \quad$ for $\mathrm{k}=0,1,2, \ldots, N$.

For instance, when considering data issued from numerical simulations the function $f$ can be associated with a numerical flow solver that enables to step forward the velocity vectors in the grid space. As the flow is three dimensional it is natural to define the system state variable as the $3 \mathrm{D}$ velocity at each point of the grid. The space dimension is $n=3 n_{g}$, and the state vector is evaluated over $N$ time steps.

One can consider now an observable defined as a vector-valued function $g(\underline{X})$, with $g: \mathbb{R}^{n} \rightarrow \mathbb{R}^{p}$, in which $p<n$. The essential idea of the Koopman analysis is that by monitoring long time series of an incomplete observable, one can characterize the behavior of the fluid dynamical system. This is based on Taken's embedding theorem [8] that assures that when the state of a system is confined to an attractor in the phase space, the topology of the attractor is preserved by the embedding. The observables of the state of a fluid flow system can be of different types [6]. In the most usual cases time series of two velocity components determined in a slice are considered. For instance, if the velocity fields are determined by means of a PIV system, the observable is a vector of size $p=2 n_{g_{2 D}}$ formed with the components of the velocity fields at the different points of the grid of size $n_{g_{2 D}}$.

The Koopman operator (or composition operator) $K_{f}$ defined with respect to a function $f: \mathbb{R}^{n} \rightarrow$ $\mathbb{R}^{n}$ is a linear operator that acts on scalar-valued functions defined on $\mathbb{R}^{n}$. In this analysis, as the function $f$ is determined by equation (11), we can drop the subindex and define the Koopman operator $K$ in the following way. If $g: \mathbb{R}^{n} \rightarrow \mathbb{R}$ then $K$ maps $g$ into a new function $K g: \mathbb{R}^{n} \rightarrow \mathbb{R}$ such that

$K g(\underline{X})=g(f(\underline{X}))$,

where $\underline{X} \in \mathbb{R}^{n}$. Formally, we consider $K: L^{2}\left(\mathbb{R}^{n}\right) \rightarrow L^{2}\left(\mathbb{R}^{n}\right)$.

One can observe that the Koopman operator steps forward the observable in time as

$K g\left(\underline{X}^{k}\right)=g\left(f\left(\underline{X}^{k}\right)\right)=g\left(\underline{X}^{k+1}\right)$.

The dynamics described by equation (11) can be analyzed through the eigenvalues and eigenvectors of $K$. Let $\lambda_{j} \in \mathbb{C}$ indicate the eigenvalues and $\varphi_{j}: \mathbb{R}^{n} \rightarrow \mathbb{C}$ the eigenfunctions of the Koopman operator, we have then

$K \varphi_{j}(\underline{X})=\lambda_{j} \varphi_{j}(\underline{X})$.

According to $[3,16]$ as $N \rightarrow \infty, K$ is an unitary operator and therefore the sequence of eigenfunctions $\varphi_{j}$ forms an orthonormal expansion basis [14]. The observables can be thus expanded as:

$g(\underline{X})=\sum_{j=1}^{\infty} b_{j} \varphi_{j}(\underline{X})$,

where $b_{j} \in \mathbb{C}$.

Now, considering as observable of the dynamic system a vector-valued function $\mathbf{g}: \mathbb{R}^{n} \rightarrow \mathbb{R}^{p}$,

$\underline{g}(\underline{X})=\left(\begin{array}{c}g_{1}(\underline{X}) \\ g_{2}(\underline{X}) \\ \ldots \\ g_{p}(\underline{X})\end{array}\right)$

where each component $g_{i}$ is a function of $\mathbb{R}^{n}$ into $\mathbb{R}$ and assuming that $g_{i}(\underline{X})$ lies in the space spanned by the basis defined by the eigenfunctions, we get the following decomposition:

$\underline{g}(\underline{X})=\left(\begin{array}{c}g_{1}(\underline{X}) \\ g_{2}(\underline{X}) \\ \cdots \\ g_{p}(\underline{X})\end{array}\right)=\left(\begin{array}{c}\sum_{j} b_{j 1} \varphi_{j}(\underline{X}) \\ \sum_{j} b_{j 2} \varphi_{j}(\underline{X}) \\ \ldots \\ \sum_{j} b_{j p} \varphi_{j}(\underline{X})\end{array}\right)$. 
This can be written more directly as

$\underline{g}(\underline{X})=\sum_{j} \underline{v}_{j} \varphi_{j}(\underline{X})$

in which $\underline{v}_{j}=\left(b_{j 1} b_{j 2} \ldots b_{j p}\right)^{T} \in \mathbb{C}^{p}$ and $\varphi_{j}(\underline{X}) \in \mathbb{C}$. The $\underline{v}_{j}$, is called the Koopman mode $j$ associated with the observable $\underline{g}$. In traditional DMD when observables are velocity fields on a slice, each component of this vector, is in correspondence with a point of the $2 \mathrm{D}$ spatial grid support of the PIV measurements. It can be determined as

$\underline{v}_{j}=<\underline{g}\left(\underline{X}^{0}\right), \varphi_{j}\left(\underline{X}^{0}\right)>_{L^{2}\left(\mathbb{R}^{n}\right)}$.

This vector, that depends on the initial condition considered, should in principle be determined only once for the first sample as the remaining samples can be expressed entirely in terms of this initial observable. This can be observed considering that

$\underline{g}\left(\underline{X}^{k}\right)=K^{k} \underline{g}\left(\underline{X}^{0}\right)=K^{k}\left(\sum_{j=1}^{\infty} \varphi_{j}\left(\underline{X}^{0}\right) \underline{v}_{j}\right)=\sum_{j=1}^{\infty} \lambda_{j}^{k} \varphi_{j}\left(\underline{X}^{0}\right) \underline{v}_{j}$.

Equation (19) indicates that the sequence of observables are decomposed into spatial structures whose temporal behavior is given by the associated eigenvalue $\lambda_{j}$. For a given eigenvalue $\lambda_{j} \in \mathbb{C}$, its phase argument is associated with the frequency of the mode $j$ whereas the modulus value can be associated with the growth rate of the mode. The growth rate of systems orbiting on an attractor is always zero and eigenvalues lie in the unit circle.

In addition, the Koopman eigenfunction $\varphi_{j}$ enables to associate to each mode an amplitude determined by $\left\|\varphi_{j}\left(\underline{X}^{0}\right) \underline{v}_{j}\right\|$. This norm constitutes a measure of the relative importance of the mode. As a consequence, it is possible to construct spectral graphs in which discrete frequencies are associated with this norm. Note that in general this kind of graph does not necessarily represent the frequencies against the associated energy of the mode.

\subsection{Numerical estimation of Eigenfunctions and Eigenvalues of Koopman operator}

Koopman modes can be calculated in principle considering the integral that defines the inner product (18). However, this integral is computationally intractable as any initial condition should be considered. Following the work of Schmid [24], we next describe other alternatives to compute these modes.

\subsubsection{Companion Matrix Method}

We consider in the following that a sequence of snapshots of the observable $\underline{g}$ is available. Let us note this sequence

$G=\left\{\underline{g}\left(\underline{x}, t_{0}\right) ; \underline{g}\left(\underline{x}, t_{1}\right) ; \underline{g}\left(\underline{x}, t_{2}\right) \ldots ; \underline{g}\left(\underline{x}, t_{N}\right)\right\}=\left\{\underline{g}^{0} ; \underline{g}^{1} ; \underline{g}^{2} ; \ldots ; \underline{g}^{N}\right\}$.

These velocity fields obtained experimentally or from numerical simulations are assumed to be sampled with a constant time step $\delta t$. Let us define a matrix gathering column by column all these snapshots:

$G_{0}^{N}=\left[\underline{g}^{0} \underline{g}^{1} \underline{g}^{2} \ldots \underline{g}^{N}\right]$.

As we are assuming dynamical systems that are orbiting on an attractor, for a very large number of snapshots $N$, it is expected that the space spanned by the vectors constituting $G$ will not change by adding new snapshots to the time series. Any new snapshot can hence be approximated as a linear combination of the previous ones

$\underline{g}^{N+1}=\sum_{m=0}^{N} s_{m} \underline{g}^{m}+\underline{r}=G_{0}^{N} \underline{s}+\underline{r}$, 
with $\underline{s}=\left(s_{0} ; s_{1} ; \ldots ; s_{N}\right)^{T}$ and where $\underline{r}$ is a residual vector belonging to the complement space spanned by $\left\{\underline{g}^{0} ; \underline{g}^{1} ; \underline{g}^{2} ; \ldots ; \underline{g}^{N}\right\}$.

Assuming a linear mapping determined by a Koopman operator $K$ linking $g^{k}$ to $g^{k+1}$, and that this mapping is approximately stationary over the sampling interval, then

$\left\{\underline{g}^{0}, \underline{g}^{1}, \ldots, \underline{g}^{N}\right\}=\left\{\underline{g}^{0}, K \underline{g}^{0}, K^{2} \underline{g}^{0}, \ldots, K^{N} \underline{g}^{0}\right\}$.

From (20) we can write:

$K G_{0}^{N}=G_{1}^{N+1}=G_{0}^{N} S+\underline{r} \underline{e}_{N+1}^{T}$,

where $\underline{e}_{N+1}$ denotes the $(N+1)$-unit vector in $\mathbb{R}^{N+1}$ and $S$ is a companion matrix of the form:

$S=\left(\begin{array}{ccccc}0 & 0 & \cdots & 0 & s_{0} \\ 1 & 0 & \cdots & 0 & s_{1} \\ 0 & 1 & \cdots & 0 & s_{2} \\ \vdots & \vdots & \ddots & \vdots & \vdots \\ 0 & 0 & \cdots & 1 & s_{N}\end{array}\right)$

It is immediate to observe that when the residual is null the matrix $S$ is similar to matrix $K$. Its eigenvalues can be used to approximate a subset of the eigenvalues of the infinite dimensional Koopman operator. The matrix $S$ can be determined by minimizing the residual $\mathbf{r}$, which is equivalent to optimally express the $N+1$-snapshot as a linear combination of $\left\{\underline{g}^{0}, \underline{g}^{1}, \ldots, \underline{g}^{N}\right\}$ in a least-square sense. Introducing in recursion $(21)$ a $Q R$-decomposition for $G_{0}^{N}$ such that $Q R=\left[\underline{g}^{0} \underline{g}^{1} \cdots \underline{g}^{N}\right]$ (where $Q$ is an orthonormal matrix) and multiplying by $R^{-1}$, we get

$K Q=Q R S R^{-1}+\underline{r} \underline{e}_{N+1}^{T} R^{-1}$.

Denoting $H=R S R^{-1}$, a Hessenberg type matrix, for small residuals we obtain the approximation

$K Q \approx Q H$,

Matrices $K$ and $H$ are similar $\left(Q^{T} K Q=H\right)$ and share the same eigenvalues (with different eigenvectors in general). Moreover, the last column of the companion matrix can be directly calculated as $\underline{s}=\left[\begin{array}{lll}s_{0} & s_{1} & s_{2} \ldots . . s_{N}\end{array}\right]^{T}=R^{-1} Q^{T} \underline{g}^{N+1}$.

\subsubsection{Preprocessing with singular value decomposition}

In some cases when working with noisy experimental data, the direct implementation of the algorithm with the companion matrix leads to an ill-conditioned system.

A more robust implementation has been proposed by Schmid [24] using a singular value decomposition of $G_{0}^{N}$

$G_{0}^{N}=U \Sigma W^{T}$,

where $U$ and $W$ are square orthonormal matrices Considering equation(21) and the given decomposition of $G_{0}^{N}$ we get

$U^{T} K U=U^{T} G_{1}^{N+1} W \Sigma^{-1}$.

Defining $U^{T} G_{1}^{N+1} W \Sigma^{-1}=\tilde{S}$, we have immediately that the companion matrix $S$ and matrix $\tilde{S}$ are similar. Hence the eigenvalues of $\tilde{S}$ approximate those of operator K. The eigenvectors $z_{i}$ of matrix $\tilde{S}$ are related with Koopman modes through:

$\varphi_{i}=U z_{i}$.

Note that for $2 \mathrm{D}$ velocity fields defined on a spatial grid of size $(N P \times N Q)$ the dimension of $G_{0}^{N}$ is $2(N P \cdot N Q) \times(N+1)$ (as the snapshot vector dimension $p=2(N P \cdot N Q))$. When $G_{0}^{N}$ is rank deficient, an incomplete SVD leads to a reduction of the size of matrix $\tilde{S}$ by keeping only the highest eigenvalues. Such a reduction not only lowers the computational load but serves also to robustify the eigenvectors estimation through a thresholding of the smallest eigenvalues. 


\section{Chronos-Koopman Spectral Approach}

In this section, we describe a formalism that allows us combining the respective advantages of POD and the Koopman spectral analysis. This technique defined as a two steps decomposition will provide a very attractive inspection technique for physical analysis of flows undergoing quasi-periodic orbits in the phase space. The combination of both decomposition techniques has been already suggested in [14] where the possibility to apply a Proper Orthogonal Decomposition subsequently to a Koopman spectral analysis was suggested. The technique proposed here is however different and relies on proceeding first to Proper Orthogonal Decomposition and then to apply a Koopman spectral analysis on the POD temporal modes. Although this might appear to be just as inversion of two computational stages, it leads to a completely different approach that leads for instance to a significant reduction of the residual space dimension generated by the spectral decomposition

Let us remark that POD technique relies intrinsically on a SVD. As explained above, Schmid's preprocessing also is based on the use of SVD but this author introduces an operation that amounts to a projection, onto a POD basis, of the linear operator used in the DMD. We propose a technique that does not project the operator but the snapshots of the velocity fields onto the POD basis. The projected operator of Schmid enables to step forward in time the snapshots of velocity field whereas the operator we consider enables to step forward in time the chronos (temporal coefficients of POD modes).

\subsection{CK Modal analysis}

In the same way as in the previous context, in order to analyse a 3D flow we assume that we have at our disposal on a spatial grid of size $(N P \times N Q)$ a large time series of $N+1$ snapshots of the velocity flow field

$$
\mathcal{H}=\left\{\underline{u}\left(\underline{x}, t_{0}\right) \underline{u}\left(\underline{x}, t_{1}\right) \underline{u}\left(\underline{x}, t_{2}\right) \ldots \underline{u}\left(\underline{x}, t_{N}\right)\right\} .
$$

Proceeding to a POD analysis of this time series, we specify as an observable of the system the vector composed by the temporal coefficients of the POD modes:

$$
\underline{A}^{k}=\left[a_{1}\left(t_{k}\right) a_{2}\left(t_{k}\right) \ldots a_{M}\left(t_{k}\right)\right]^{T} \text { for } k=0,1,2, \ldots, N .
$$

The size $M$ of this vector is at most the total number of snapshots $N+1$ if all the POD modes are retained in the analysis. We can then construct the sequence of observables

$G=\left\{\underline{A}^{0} ; \underline{A}^{1} ; \underline{A}^{2} ; \ldots ; \underline{A}^{N}\right\}$.

Performing a spectral analysis of this observable and considering equation (19) we can write :

$\underline{A}^{k}=K^{k} \underline{A}^{0}=\sum_{j=1}^{\infty} \lambda_{j}^{k} \varphi_{j}\left(\underline{A}^{0}\right) \underline{V_{j}}=\sum_{j=1}^{\infty} e^{i\left(2 \pi k \Omega_{j}\right)} \varphi_{j}\left(\underline{A}^{0}\right) \underline{V_{j}}$.

In practice the upper limit of summation is bounded by the total number of snapshots. Modes $V_{j}$ are vectors of size $M$ such that each component corresponds to a POD mode. We identify these vectors as the Chronos-Koopman (CK) modes.

Each one of these CK modes is associated with a single frequency $\Omega_{j}$, that can be obtained in the same way as described in the traditional spectral decomposition of the Koopman operator. In practice we rely on the technique based on the companion matrix without the SVD preprocessing step which appears to be unnecessary in this case.

It is worthy to note that $\underline{s}$, the last column of the companion matrix, can be calculated through a least square technique as

$\underline{s}=\left[\begin{array}{llll}s_{0} & s_{1} & s_{2} \ldots . . s_{N}\end{array}\right]^{T}=\left(G_{0}^{N^{T}} G_{0}^{N}\right)^{-1} G_{0}^{N^{T}} \underline{g}^{N+1}$. 
The observable at time $t_{k}$ with a CK analysis $\underline{A}^{k}$ is determined as a projection of the velocity field at this time onto POD spatial modes (topos), $\underline{\Phi}^{T} u\left(x, t_{k}\right)=A^{k}$

Introducing this last expression in eq. (26) (and recalling that $\underline{\Phi}^{T} \underline{\Phi}=I$ where $I$ denotes the identity matrix) we can see straightforwardly that the last column of the companion matrix of traditional DMD (taking as observable the velocity field) or CK approach (taking as observable the temporal coefficients of POD) are both the same. Thus the eigenvalues computed with both approaches are identical.

Each of the CK modes $V_{j}$ is associated with a spatial structure in the physical domain through the POD spatial modes $\underline{\Phi}_{i}(\underline{x})$. Defining the coefficient associated with the POD mode $r$ as $p_{j}^{r}=V_{j}(r) \varphi_{j}(0)$, the spatial representation reads:

$\underline{\Psi}_{j}(\underline{x})=\sum_{r=1}^{M} \underline{\Phi}_{r}(\underline{x}) p_{j}^{r}$.

These spatial structures (or spatial modes), obtained as a summation of the spatial modes of the POD weighted by the amplitudes of the CK modes are identical to the Koopman spatial modes $v_{j}$ that would be obtained with a DMD analysis performed directly on the snapshots of the velocity field. With this approach we can thus recover all the significant results obtained when performing a traditional DMD analysis from snapshots of the velocity fields.

However, compared to the traditional DMD approach, the technique we propose presents several advantages:

- In traditional DMD the evolution operator has a dimension that is bounded by the square of the snapshot vector dimension $(p)$. In the usual case, in which $N$, the number of snapshots, is lower than the dimension of the snapshot vector, the number of eigenvalues that can be estimated is only $N-1$. As the evolution operator has a maximum number of eigenvalues equal to the snapshot vector size, an important amount of modes of the decomposition $(p-N)$ will likely be missing . The CKS approach we propose considers an evolution operator with a maximum dimension $(N \times N)$. The number of eigenvalues that is possible to approximate is $N-1$. The decomposition is expected to be almost complete as only one mode may be missing .

- The implementation of spectral methods based on Koopman's operator relies on the hypothesis that a basis can be formed with $N-1$ snapshots such that any new snapshots can be expressed in terms of the preceding ones. When $N-1$ is much lower than the snapshot vector size, the basis is far to be complete, and the residual vector $\underline{r}$ will lie in a high dimensional space $(p-N)$. In the CKS approach a vector of observable of size $N$ may be approximated with a basis formed by the $N-1$ preceding vectors. If all these vectors are linearly independent then the basis is almost complete. In this case, the subspace spanned by the basis is more appropriate and the residual lies only in a one dimensional space. Obviously similar conditions can be attained for both approaches by enlarging the dataset. This is however paid by an unnecessary higher computational cost that may become prohibitive in cases where accurate flow descriptions requires the use of fine spatial-temporal grids.

- As further discussed in the following section, the amplitude of DMD modes have an easy physical interpretation only in the ideal case when all eigenvalues are complex roots of 1 . The mode amplitude of our approach enables to construct a suitable power spectrum representation.

\subsubsection{Energy Spectrum}

Considering the traditional algorithm of DMD, for unitary eigenvalues $\left|\lambda_{j}\right|=1$, it is easy to show that the power spectral density is recovered by considering the $\mathcal{L}_{2}$-norm of the Koopman modes associated with each frequency channel. When $\left|\lambda_{j}\right|$ is either smaller or greater than one, equivalence between power spectral density and Koopman mode norm does not hold anymore. This is due to the fact that the modes are non orthogonal, and, that when $\left|\lambda_{j}\right|<1$ (resp. $\left|\lambda_{j}\right|>1$ ) their weight is decreasing (resp. 
increasing) along time. To recover the power spectral density in this case one natural idea would be to weight the norm of the Koopman mode by a coefficient of the form

$\frac{1}{N} \sum_{j=1}^{N}\left|\lambda_{j}\right|^{2}$,

that would write

$\frac{1}{T} \int_{0}^{T} \mathrm{e}^{2 \ln \left|\lambda_{j}\right| t} \mathrm{~d} t$

in the continuous form, such as to take into account the mean weight of the mode over the observation time range $T=(N) \delta t$. Still, this would not be equivalent to the power spectral density, as a straightforward calculus would show.

With the technique we propose each CK mode can be related to an energy content. Denoting

$m_{r}=\sum_{j=1}^{N}\left|p_{j}^{r}\right|^{2}$,

and

$Z=\left[\underline{A}^{0} \underline{A}^{1} \ldots \underline{A}^{N-1}\right]$

it is possible to express $Z$ as a product of two matrices, $Z=\Xi T$ where

$\Xi=\left(\begin{array}{ccccc}p_{1}^{1} & p_{2}^{1} & p_{3}^{1} & \cdots & p_{N}^{1} \\ p_{1}^{2} & p_{2}^{2} & p_{3}^{2} & \cdots & p_{N}^{2} \\ p_{1}^{3} & p_{2}^{3} & p_{3}^{3} & \cdots & p_{N}^{3} \\ \vdots & \vdots & \ddots & \vdots & \vdots \\ p_{1}^{M} & p_{2}^{M} & p_{3}^{N} & \cdots & p_{N}^{M}\end{array}\right)$

and

$T=\left(\begin{array}{ccccc}1 & \lambda_{1} & \lambda_{1}^{2} & \cdots & \lambda_{1}^{N-1} \\ 1 & \lambda_{2} & \lambda_{2}^{2} & \cdots & \lambda_{2}^{N-1} \\ \vdots & \vdots & \ddots & \vdots & \vdots \\ 1 & \lambda_{N} & \lambda_{N}^{2} & \cdots & \lambda_{N}^{N-1}\end{array}\right)$

is a Vandermonde matrix.

Considering the product $Z \overline{Z^{T}}=\Xi T \overline{(\Xi T)^{T}}$, and provided that all eigenvalues are complex N-th roots of 1 , then $Z \overline{Z^{T}}=N \Xi I \overline{\Xi^{T}}$, where $I$ denotes the identity matrix.

It is a consequence of this equation that the kinetic energy of any r-th POD mode will satisfy the following identity $\left\langle a_{r}^{2}\right\rangle=m_{r}$.

This identity indicates that the energy of a given POD mode receives a contribution of the modes associated with the different eigenvalues (frequency channels) determined by the square of the mode amplitude $p_{j}^{r}$. The energy associated with a j-th frequency channel when the eigenvalues map perfectly in the unit circle can be expressed as

$E_{j}=\sum_{r=1}^{M}\left|p_{j}^{r}\right|^{2}$ 
However, in general the restriction imposed to the mapping expressed above is not satisfied (for instance due to the existence of evanescent modes) and the identity $\left\langle a_{r}^{2}\right\rangle=m_{r}$ does not hold anymore. In a more general case, it is possible to consider that the energy of a given r-th POD mode $\left\langle a_{r}^{2}\right\rangle$ is distributed among the different frequency channels $j$ with a proportionality factor equal to $\left|p_{j}{ }^{r}\right|^{2} / m_{r}$.

The energy content of the CK mode can then be formalized as the sum

$E_{j}=\sum_{r=1}^{M}\left[1 / m_{r}\right]\left|p_{j}^{r}\right|^{2}\left\langle a_{r}^{2}\right\rangle$

The summation of the energy content of all CK modes allows us recovering the total fluctuating kinetic energy of the flow. Note that this expression reduces to equation (32) in the ideal case when the restriction of the mapping is satisfied.

It is sometimes convenient to normalize the energy expression (33) as follows

$\tilde{E}_{j}=\left[1 / \sum_{j=0}^{N} E_{j}\right]\left\{\sum_{r=1}^{M}\left[1 / m_{r}\right]\left|p_{j}^{r}\right|^{2}\left\langle a_{r}^{2}\right\rangle\right\}$

The approach proposed here enables thus to categorize the modes $V_{j}$ with an energy criterion. This gives us the possibility to identify modes that concentrate higher energy levels, and to determine the respective contribution of each mode to the total fluctuating energy of the flow.

An inspection of CK modes enables to detect the coupling between dynamical structures of high and low energy which are sharing a common frequency. In many cases the structures with higher levels of energy exhibit larger spatial structures and finer scales can be associated with structures of lower energy. It is expected that suitable analysis of these modes may provide essential elements for the modeling of interacting structures of different size. Although, beyond of the scope of this article, characteristic features enabling to propose sub-grid models could be for instance unveiled from such a data analysis.

\subsubsection{CK Spectral analysis and Discrete Fourier transform}

As we are considering dynamical systems with attractors, Koopman modes may be calculated by harmonic averages, which for finite-time datasets reduce to discrete Fourier transforms [6, 14].

We can analyse the series $\left\{\underline{A}^{0}, \underline{A}^{1}, \ldots, \underline{A}^{N}\right\}$ and the DFT of this sequence, denoted as $\left\{\underline{B}_{0}, \underline{B}_{1}, \ldots, \underline{B}_{N}\right\}$, and defined by

$\underline{B}_{j}=\lim _{N \rightarrow \infty} \frac{1}{(N+1)} \sum_{k=0}^{N} \underline{A}^{k} e^{-i \frac{2 \pi k}{N+1} j} \quad$ for $\mathrm{j}=0, \ldots, N$.

As

$\underline{A}^{k}=\sum_{l=0}^{N} \lambda_{l}^{k} \underline{v_{l}}$

we get

$\underline{B}_{j}=\lim _{N \rightarrow \infty} \frac{1}{(N+1)} \sum_{k=0}^{N} \sum_{l=0}^{N} \lambda_{l}^{k} \underline{v}_{l} e^{-i \frac{2 \pi k}{N+1} j}=\lim _{N \rightarrow \infty} \frac{1}{(N+1)} \sum_{l=0}^{N} \underline{v}_{l} \sum_{k=0}^{N} \lambda_{l}^{k} e^{-i \frac{2 \pi k}{N+1} j}$. 
provided $\left|\lambda_{l}\right|=1$, and particularly considering $\lambda_{l}=e^{i \frac{2 \pi n_{l}}{N+1}}$, we obtain

$\underline{B}_{j}=\lim _{N \rightarrow \infty} \sum_{l=0}^{N} \underline{v}_{l} \delta\left(n_{l}-j\right)=\underline{v}_{n_{l}}$

The DFT coefficients in the expansion are regularly distributed along the frequency domain and taking into account [16] the same regular spacing should be observed for the Koopman's modes in the energy spectrum. Thus, the number of Fourier and Koopman's mode are the same and under the hypothesis considered for a given frequency their amplitude (or energy spectrum) should be also identical up to a constant.

In practice however the eigenvalues do not lie exactly on the unit circle. This can be related for instance to small fluctuations in experimental conditions that lead to damping modes in the interior of the unit circle. The numerical errors and also the approximations of the algorithms used to estimate the eigenvalues and eigenvectors of Koopman operator may also introduce a discrepancy between the estimates and the unit circle. As the cyclic character of Koopman operator indicates that eigenvalues should be equispaced, errors in eigenvalues estimation may become evident through an irregular angular spacing of the eigenvalues on the complex plane. Also, for finite datasets ( $\mathrm{N}$ finite), the state of the system starting at time 0 will not necesarilly agree with the sate corresponding at the instant of the last snapshot $\mathrm{N}$ and also a repetition of the trajectory observed in the first sequence do noy necesarilly will be reproduced by the following sequences of $\mathrm{N}$ snapshots. The implementation of a discrete Fourier analysis over a sequence assumes implicitely such a periodicity. Diverse strategies can be proposed to overcome this limitation but is important to signal here that this kind of requirement is not present in a Koopman analysis. Thus in practice the correspondence between results issued from a Fourier or Koopman decomposition has to be carefully analysed in each case. Particularly, special attention should be paid when associating the values of mode amplitude of traditional Koopman's analysis to energy content of the mode.

\subsubsection{Graphical Interpretation of Chronos-Koopman spectral decomposition}

A clear interpretation of the procedure proposed and the links between POD and spectral modes can be easily pictured by considering the following scheme. Let us construct a discrete 3D graph (see Figure 1) in the following way. In one axis we represent frequencies. As each CK mode $j$ has a single frequency $w_{j}$ we can include a slice containing the mode $V_{j}$ weighted by $\varphi_{j}(0)$ in each one of these coordinates. Then we have as coordinate for the second axis of the graph the POD mode number and for the third axis $p_{j}^{r}=V_{j}(r) \varphi_{j}(0)$. When making intersection of this graph with planes with a constant POD mode number $r$, we get a spectrum showing the contribution of the temporal mode along the frequency domain $w_{j}$.

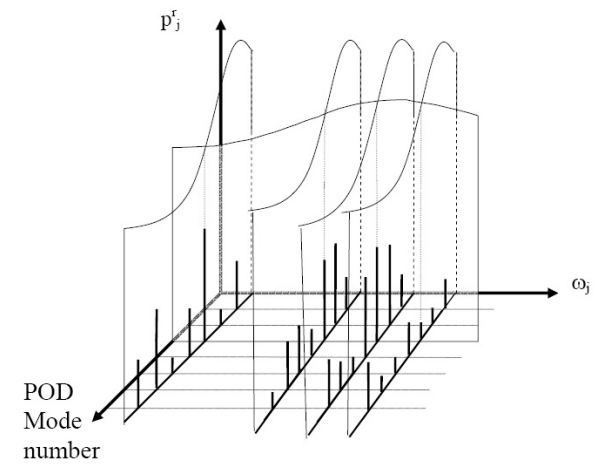

Fig. 1 Schematic representation of Chronos-Koopman Spectral Decomposition. 
It is of interest to recall that the spatial structures of traditional Koopman modes associated with a given frequency $w_{j}$ can be obtained in this case considering the different topos and the vertical coordinate of this graph $p_{j}^{r}$ as:

$\underline{\psi}_{j}(x)=\sum_{r=1}^{M} \underline{\phi}_{r}(x) p_{j}^{r}$.

\subsection{CK Reduced Order Dynamical Modeling}

In several applications, for forecast or analysis purposes, it is primordial to devise dynamical models that allow reconstructing accurately the velocity fields not only at the snapshot instants but also at intermediate or future instants. For the particular case of dynamical system orbiting around attractors, and using the CK decomposition we have:

$\underline{A}^{k}=\sum_{j=1}^{N} \lambda_{j}^{k} \varphi_{j}\left(A^{0}\right) \underline{V_{j}}$

where $k$ corresponds to the instant at which one wishes to get an interpolated or forecasted chronos value. The value of $k$ to be considered is determined by the instant at which model prediction is required and time scale is determined by the time step between snapshots. Thus the dynamics of chronos can be described as a summation along the different frequencies arising from the CK decomposition. Note that this gives an equivalent representation as the one would expect integrating equation (9). However it is important to outline that neither the precise form of the POD-Galerkin system, nor any specific assumption on boundary conditions on pressure has to be settled with this approach. As spatial POD modes are available, the reconstruction of velocity fields or eventually derived quantities such as vorticity for any time is direct.

Hence to describe the dynamics of the flow, one can propose a CK ROM that is formed as a superposition of all the CK modes. A further reduction of the system can obtained keeping only a set of selected CK modes chosen based on an energetic threshold criteria.

Obviously predictions at intermediate states are likely to be accurate only if sampling enables to construct a model that includes all modes with significant energy (that assure that no aliasing problem could appear) and predictions at times larger than the learning interval will be reliable provided experimental conditions are kept identical. It should be mentioned that this modeling requires eigenvalues that fit almost perfectly the unit circle. The system otherwise diverges or vanishes. On long time periods, when discrepancies to the unit circle occur, corrections of outliers are possible. However a general criterion is difficult to devise.

\section{Description of the experimental database (cylinder wake)}

In order to assess the performances of the method proposed, we have constituted a benchmark composed of experimental PIV data. The experimental data concerns a wake of a circular cylinder at Reynolds 3900 . The flow considered is three dimensional with two fundamental frequencies. $f_{V S}$ is associated with Karman vortex shedding (VS) and the second one $f_{K H}$ corresponds to the instability of the separated shear layer, so called Kelvin-Helmoltz instability. Results are shown using time series of velocity field obtained in one plane perpendicular to the cylinder axis.

The wake behind a circular cylinder has been generated in the IRSTEA(Cemagref) wind tunnel. Experimental conditions are coincident with the same as those described in [9].

The free-stream velocity has been adjusted at $1.82 \mathrm{~m} / \mathrm{s}$ so that the Reynolds number be 3900 (Reynolds number based on the free stream velocity $U e$, the kinematic viscosity $\nu$ and the diameter of the circular cylinder $D=32 \mathrm{~mm}$ ). For this Reynolds number, the flow is relatively well documented in the literature $[25,28,30]$ and the Strouhal number $S t$ is 0.208 (thus in our case $f_{V S}$, vortex shedding frequency of the von Karman street, is about $11.8 \mathrm{~Hz}$ ). 
Time-resolved PIV experiments giving two in-plane velocity components in a slice have been carried out with Nd-YAG laser and a CMOS camera with $50-3000 \mathrm{~Hz}$ acquisition frequency range for $1024 \times 1024 p x$ image size.

The measurement area was located just behind the circular cylinder, in the plane lying at mid cylinder axis. The camera was located perpendicularly to the laser sheet at a distance of $60 \mathrm{~cm}$ and the resulting field of view was about $3 D \times 3 D$. Sequences of 3072 successive image pairs (equivalent to the 8 Go buffer memory of the fast camera) has been obtained with a $500 \mathrm{~Hz}$ acquisition rate $(\approx 40$ snapshots by vortex shedding). The time interval between two pulses was $250 \mu \mathrm{s}$.

The PIV records have been analyzed through a cross-correlation technique implemented with a FastFourier-Transform algorithm in a multi-grid process with 5 iterations and sub-pixel shift $(1 \times 128 \times 128$, $1 \times 64 \times 64$ and $3 \times 32 \times 32$ pixels) and $50 \%$ overlapping. The cross-correlation peaks were fit with a Gaussian function on 3 pixels. Erroneous velocity vectors have been identified by a median filter and replaced by their local mean value. To give an idea of the corresponding experimental accuracy, the particle displacement range is about -1 to +5 pixels (with an error less than 0.2 pixel on the instantaneous displacement) and less than $0.1 \%$ of the velocity vectors are erroneous.

Figure 2 illustrates a snapshot of the span wise vorticity $\omega_{z}$ (dimensionless with $U e$ and $\nu$ ). Spatial resolution for $(32 \times 32$ pixels final window size and $50 \%$ overlapping $)$ act as a low-pass filter but the noise level is emphasized by the derivative scheme.

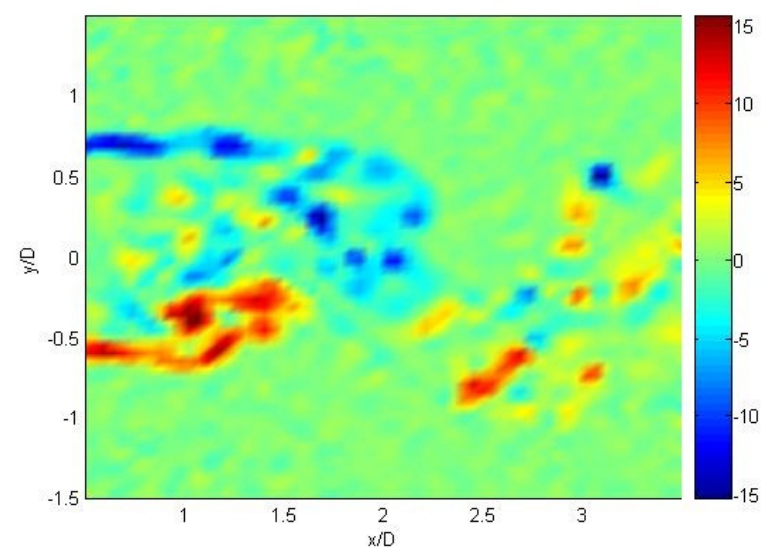

Fig. 2 Vorticity field of a snapshots of a PIV sequence.(Case I)

The spanwise vorticity $\omega_{z}$ highlights the two shear layers on both sides of the cylinder. In this image whereas one of the two shear layers is almost straight up to $x / D=2$, the second one rolls up to form a primary vortex. Small vortices resulting from the instability of this shear layers accumulate in this primary large scale structure. This scenario is observed alternatively in the two shear layers and at different stages of their evolution in the instantaneous fields. A comparison of such kind of experimental data with numerical simulations results has been already published [21]. The threedimensional instantaneous flow fields obtained by numerical simulations, which are not illustrated here, show clearly the fully three-dimensional character of the dynamics. Representing a slice of these results it can be observed a more complex pattern of vorticity with numerous vortex filaments that cannot be observed in the experiments because of the spatial resolution here adopted.

A time-resolved sequence of 3072 successive snapshots of velocity fields is directly used as data. The sampling enables to obtain approximatively 80 snapshots by vortex shedding period. Results obtained by Dong et al [25] show that the signature of vortex shedding remains a long time in the wake whereas the influence of high frequency shear layer vortices is confined to the very near wake of the cylinder. Thus the characteristic peak of the shear layer instabilities in flow spectra vanishes quite soon downstream. To undertake a spectral analysis that brings to surface the modes associated with the shear layer instabilities it is convenient to analyse a reduced window of the field of view. In this work we selected the upper left quarter of the field referred as case II to distinguish from case I 
Table 1 Characteristics of the available velocity fields, $N$ :number of snapshots, nc:number of cycles of vortex shedding, $n_{x}, n_{y}$ number of PIV grid points in both directions.

\begin{tabular}{|l|llll|}
\hline & $N$ & $T[n c]$ & $L_{x} \times L_{y}$ & $n_{x} \times n_{y}$ \\
\hline Case I (VS) & 3072 & 72 & $3 D \times 3 D$ & $64 \times 64$ \\
Case II (KH) & 2048 & 72 & $1.5 D \times 1.5 D$ & $32 \times 32$ \\
\hline
\end{tabular}

that involves the whole picture. Table 1 summarizes the main characteristics of the velocity fields used directly from the PIV database.

\section{CK Analysis of the cylinder wake data}

A Proper Orthogonal Decomposition was performed with the snapshot method [26] using the sequences of the PIV measurements. The analysis has been carried out subtracting from the data the mean (timeaverage of the sequence) velocity field of the flow. The norm of the mean velocity field is shown in Figure 3. It is comparable to those observed by other researchers (see for instance [25]) for the same type of flows.

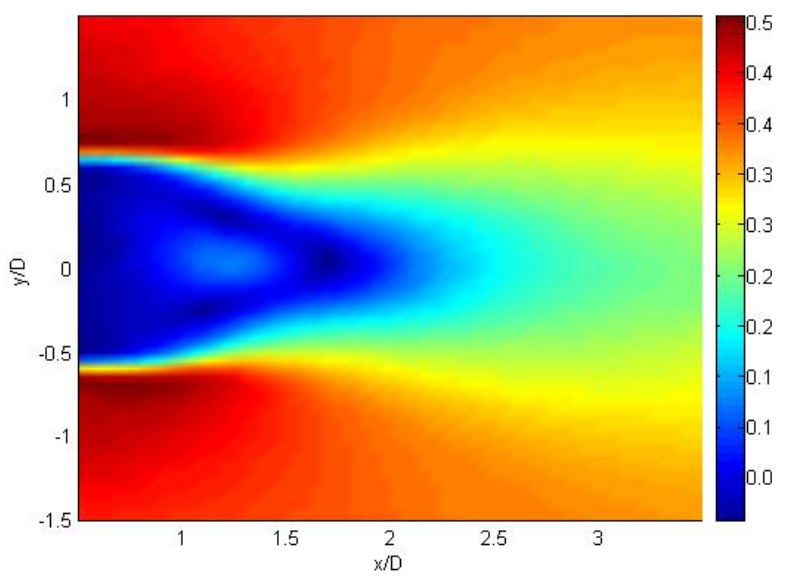

Fig. 3 Velocity norm of the mean flow.

Figure 4 presents the distribution of the cumulative fluctuating kinetic energy with respect to the number of modes. The first two modes are the principal modes of the flow. Modes 1 and 2 represent $62 \%$ of the total fluctuating kinetic energy, whereas the first 32 modes concentrate $84 \%$. An important amount of energy is thus concentrated in the first two modes and the remaining energy is more scattered distributed. This can be explained by the relatively "high" Reynolds number of the considered flow on the other modes.

On Figure 5a) and 5b) we display the spatial modes associated with the first two modes. In these figures we represent as a color map the velocity norm. The graph enables to show that these modes exhibit symmetric and regular structures of relatively large size.

The first and second modes correspond to the structure of the von Karman street vortices and to their advection downstream. This can be observed through the Power spectrum of the temporal coefficients of mode 1 and 2 obtained by DFT. These spectra are shown in Figure 6 and illustrate that these modes exhibit peaks close to the natural shedding frequency $(\sim 11.5 \mathrm{~Hz})$.

Also we performed a traditional DMD analysis (Schmid's method) for the time series of the velocity fields. Observables considered in this analysis are the components of the velocity vector in the different points of the PIV grid. To allow comparison with POD Koopman analysis of next section, we remove from the snapshots data the mean velocity flow of Figure 3. The spectral decomposition is thus done just considering the fluctuating part of the field. Results of such analysis for case I and case II are 


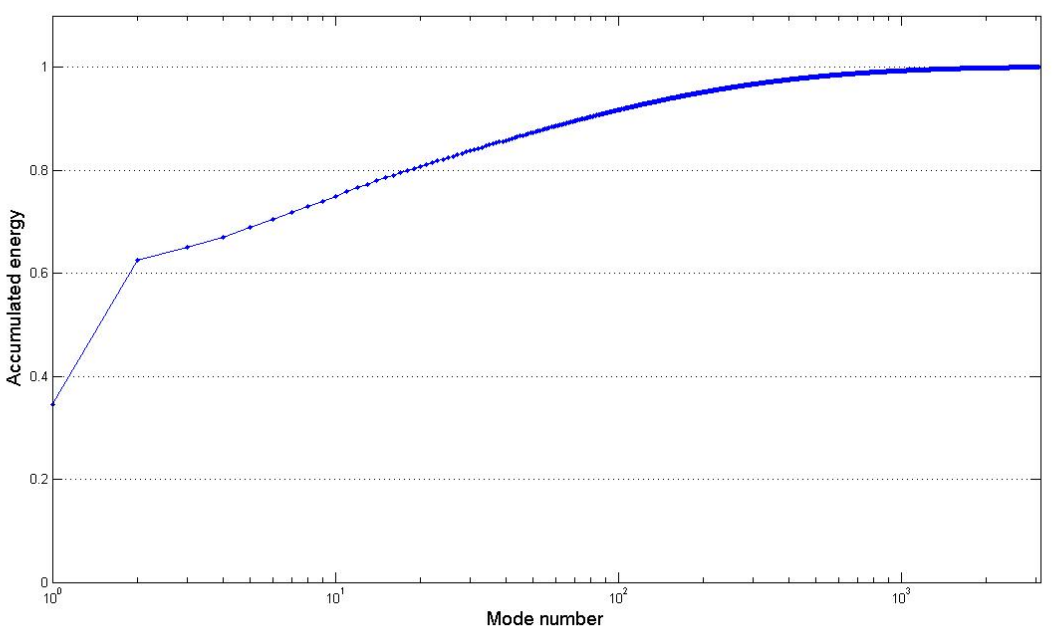

Fig. 4 Cumulative fluctuating energy distribution with respect to the number of POD modes. Values are normalized with the value of the total fluctuating energy.
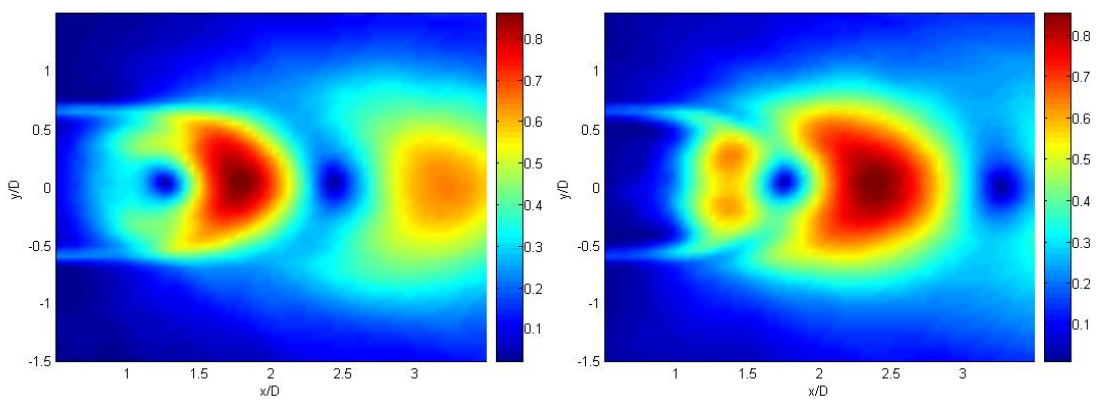

Fig. 5 Spatial modes of POD (Velocity norm)- a)Mode 1, b)Mode 2.

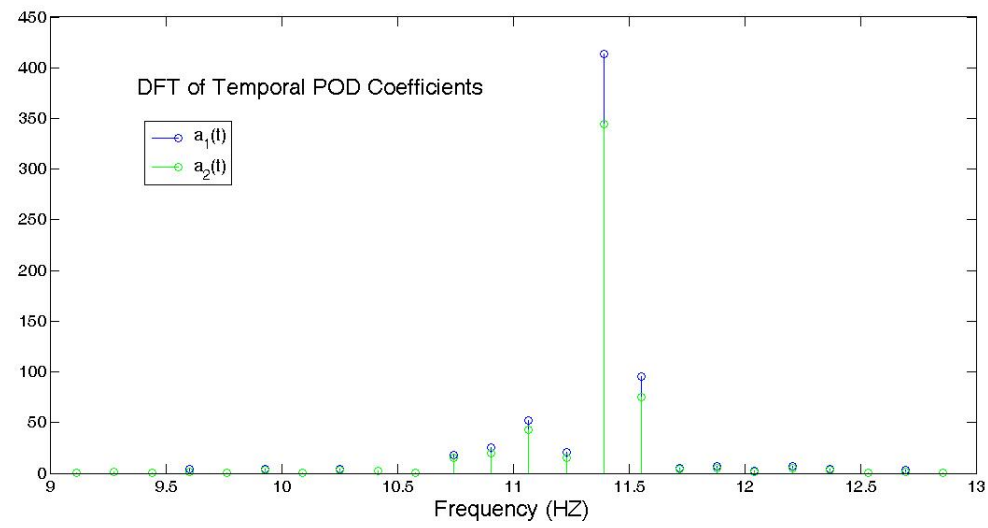

Fig. 6 Frequency spectrum of temporal coefficients of Mode 1 and 2 of POD obtained by Fourier analysis (Case I). 
shown in terms of the map of eigenvalues (Figure 7a) and 7b)) and spectra (Figure 9 and 10). The vertical coordinate of the spectra represents the norm $\left\|v_{j}\right\|$.
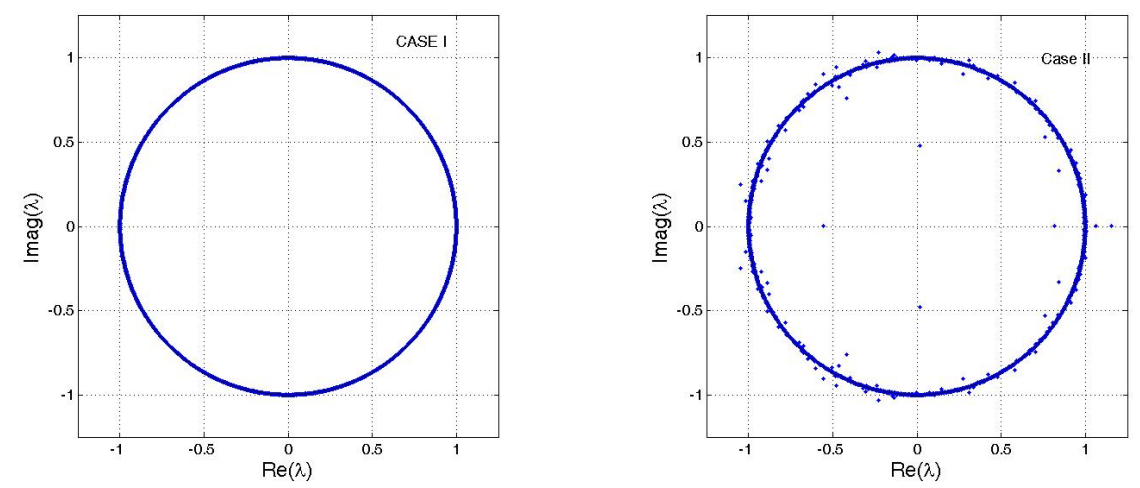

Fig. 7 Map of eigenvalues of DMD modes. a)Case I, b)Case II.

As we can observe, for the case I the eigenvalues align remarkably well on the circumference of the unit circle. A very reduced dispersion occurs and the points that are in the exterior of the circle lie at a distance from the center lower than 1.0017. For case II few outliers inside or outside of the unit circle can be observed.

Spectra for case I show a sharp peak corresponding to the vortex shedding frequency. Other peaks related to second and third harmonic of this frequency are also visible. In the spectra of case II, the peak associated with vortex shedding is much less important. In this graph and contrary to Dong et al work, we do not identify a broadband spectrum with a peak close to $7.3 \times f_{V S}$ that could be associated with vortex formation in the shear layer. Besides, data do not fit very well with the $-5 / 3$ slope that should be observed in the inertial range. Next we present some results obtained using the CKS procedure we have detailed previously.

\subsection{Chronos-Koopman spectral analysis of a wake flow}

We recall that for the CK analysis the observables are defined as the temporal coefficients of the POD modes. As discussed previously eigenvalues of CK approach and those of DMD should agree, so the mapping of CK eigenvalues correspond to those of Figure 7. On figure 8 we also show for case I and for the first two temporal coefficients, the frequency spectrum of mode amplitudes of a CK analysis (respectively $\left|p_{j}^{1}\right|^{2}$ and $\left|p_{j}^{2}\right|^{2}$ ) and the spectrum of mode amplitudes of a DFT analysis. As we can see in this case we study, similar results are obtained with both approaches.

\subsubsection{Energy Spectra of Chronos-Koopman modes}

We show in Figure 11a) and b) the normalized energy spectrum of Chronos-Koopman modes for the case I and II respectively. The graph is constructed by representing, for the different frequencies of each Chronos-Koopman mode, the associated fluctuating kinetic modal energy as proposed in equation (34). Frequencies are determined considering the argument of the different eigenvalues. The horizontal and vertical scale for the graph represented in Figure 11 are logarithmic. Because the eigenvalues are organized in conjugated pairs, the spectrum is symmetric with respect to vertical axis at null frequency. In these graphs only the positive frequencies side is shown and contribution of modes with negative frequencies is incorporated in the corresponding positive frequency mode. Conjugate pairs of eigenvalues have associated conjugate pairs of eigenvectors. Then the modal energy content for each positive frequency is thus doubled because of the contribution of the negative counterpart. Continuous line with a slope equal to $-5 / 3$ are represented to identify the inertial range of the spectrum.

For frequencies close to the vortex shedding frequency, we can observe in Figure 11a) a group of modes that concentrate the higher peaks of energy. The maximum of this group occurs for a frequency 

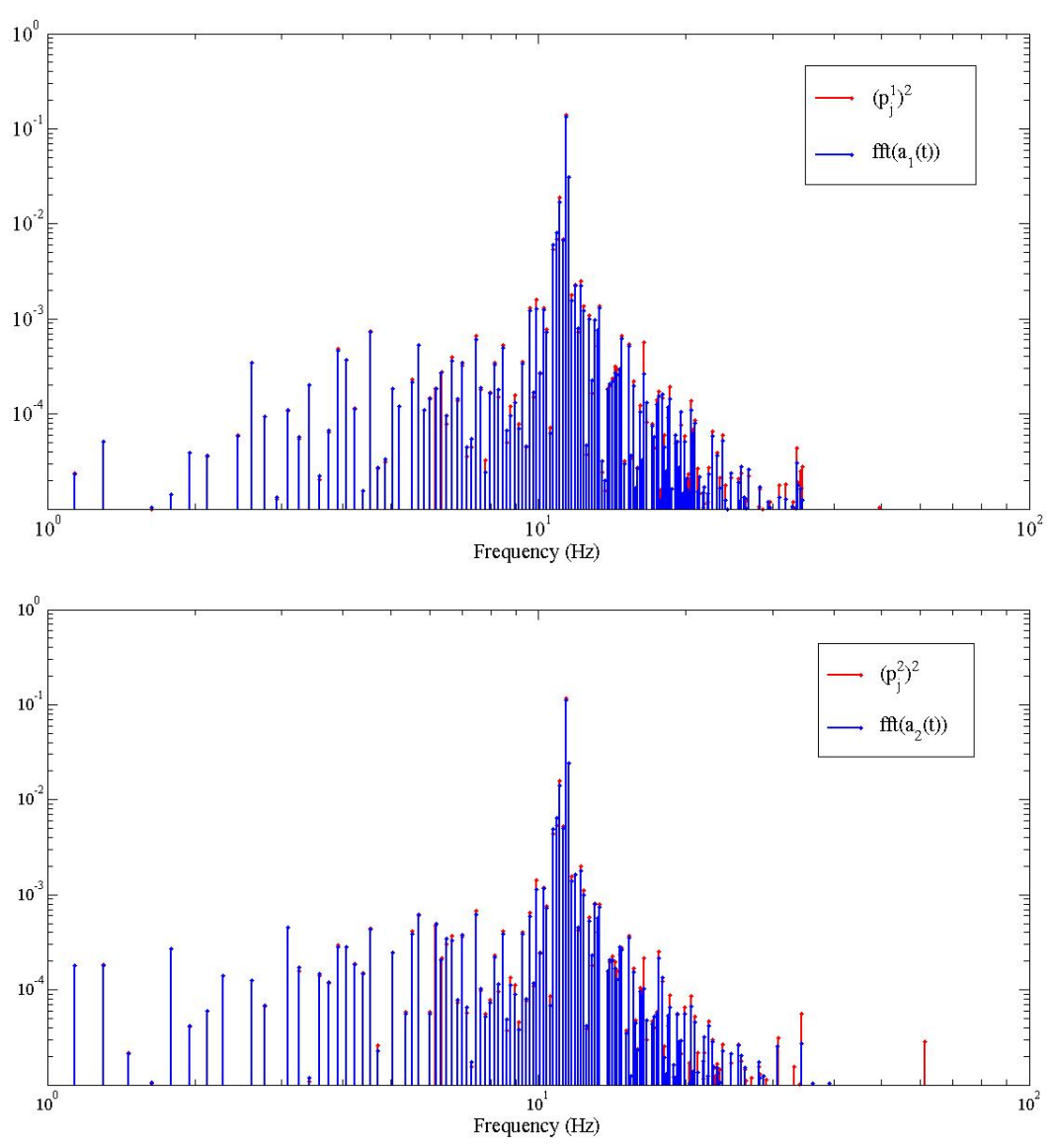

Fig. 8 Comparison of modes amplitudes of DFT and CK analysis.

of $11.39 \mathrm{~Hz}$. Smaller peaks exist in proximity of this peak but this takes place in a narrow band. This first group of peaks concentrate 61.5 percent of the total fluctuating energy. This value is quite similar to the energy contained in the first two modes of POD whose predominant frequencies determined by DFT are close to $11.6 \mathrm{~Hz}$. Pronounced group of energy peaks are also found at frequencies close to first harmonic and second harmonic of the vortex shedding frequency. The graph of Figure 11b is less scattered at the higher frequencies. These graphs show the peak associated with vortex shedding but also helps in clearly identifying the inertial subrange. This range has an extension of almost one decade in agreement with works of other researchers $[21,25]$. The analysis performed with a reduced window reveal the relative broadband spectrum corresponding to the shear layer instabilities. This occurs at frequencies close to these documented in the literature for this flow $\left(f_{K H}=7.33 \times f_{V S}=83.5 H z\right)$ [25]. The broadband nature of shear layer vortices has been attributed in different works [2,25] to temporal variations of velocity scale owing to Karman vortex formation and also to the oscillations of the separation point. A comparison of this graph with the one of Figure $9 \mathrm{~b}$ emphasizes the interest of representing energy frequency spectra (POD Koopman spectral approach) instead of mode amplitude frequency spectra (DMD approach).

\subsubsection{Chronos-Koopman modes at the frequency of instabilities $f_{V S}$ and $f_{K H}$}

We represent on Figure 12 the CK modes that correspond to the frequencies of the vortex shedding and on Figure 13 those related to shear layer instability. This representation of the modes allows to visualize how are coupled coarser structures associated with POD modes at low frequencies and finer structures associated with POD modes at higher frequencies. As can be observed the number of POD modes coupled by VS are mainly those that have associated larger energies (coarser scales). For the component number (or POD mode) whose number exceeds 380 the coupling is very reduced. For these 

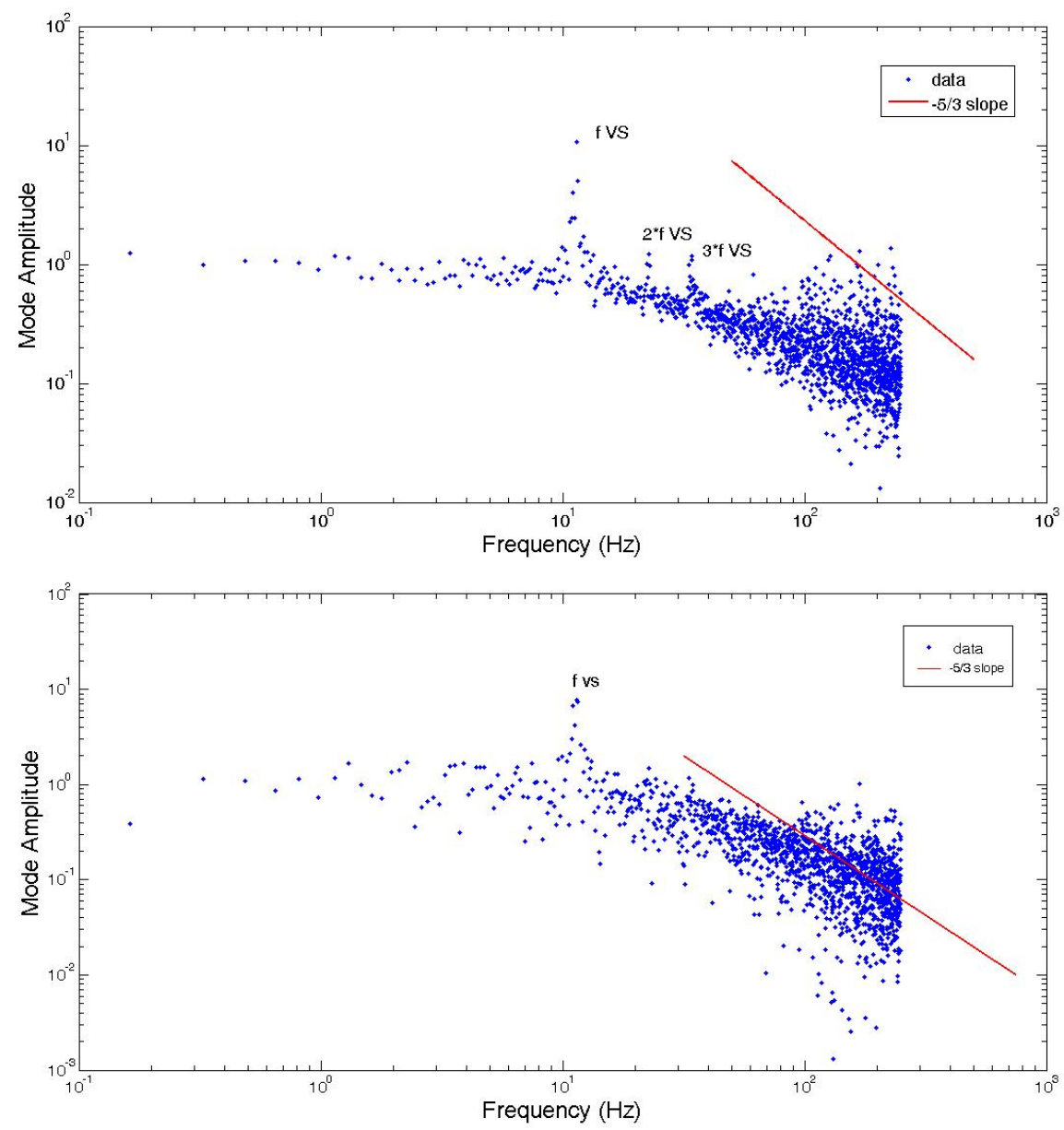

Fig. 9 Frequency spectrum obtained by DMD using as observables the velocity field components: Case I a)Using Companion matrix algorithm, b) Using the SVD preprocessing [22].

components the values of $p_{j}^{r}$ are lower than 1 per cent of the maximum value observed for component 1 and 2. It can also be seen that in this CK mode a pairing of component appears, this means for instance that amplitudes for component 1 and 2 are equal as it happens for the successive pairs.

Shear layer instability modes exhibit larger number of interacting POD modes involving more finer structures. The coupling can be perceived for modes number much greater than 1000. This graph illustrates the difficulties that have to face traditional POD-ROM formulated with a few number of modes to suitably recover the flow dynamics .

\subsubsection{Spatial Modes with associated frequency $f_{V S}$ and $f_{K H}$}

As already signaled, it is possible to recover the spatial configuration of velocity fields associated with the different CK modes. It is worth mentioning that these modes coincide with the spatial mode a traditional DMD analysis would have given. Due to the three dimensional character of the flow, we cannot directly associate the spatial structures to global modes of instabilities. Being the data obtained within a slice of the flow, they just represent the spatial structure of the observable associated with each CK mode. Figure 14 and Figure 15 show the spatial modes for the case of the modes with peaks in frequencies of $f_{V S}$ and $f_{K H}$ respectively.

We can observe that the modes lying in the narrow band frequency associated with the Karman vortex shedding exhibit a relative large and organized structure. This agrees with the results of Ma et al [28] who observed that most of the contribution to the fluctuating energy comes from the largest organized flow structures. In particular, the mode associated with the peak of the spectrum, 

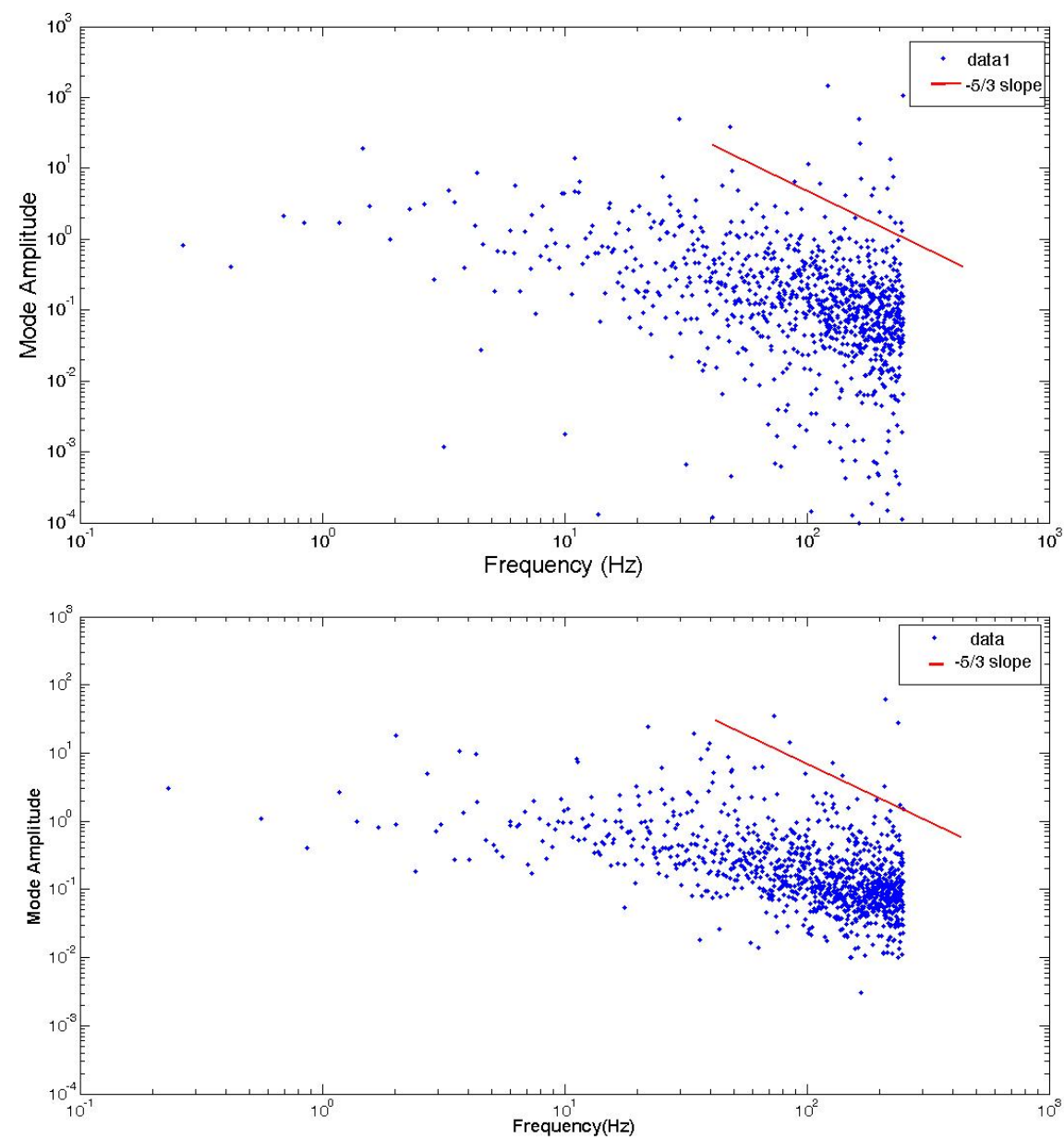

Fig. 10 Frequency spectrum obtained by DMD using as observables the velocity field components:Case II.a)Using Companion matrix algorithm, b) Using the SVD preprocessing [22] .

represented on Figure 14, shows a great similarity with the first spatial POD mode of Figure(5). This can be associated with the relative monochromatic character of the most energetic modes of POD as pictured the results of Figure 6. Modes corresponding to the broadband spectrum of shear layer instabilities are less organized and exhibit smaller structures. Typical vortex of the shear layer are of the order of $\mathrm{D} / 4$.

\subsection{CK Model Reduction}

As outlined previously, our approach enables to obtain values of the chronos at the observation instants and also at intermediate or future instants.

The chronos values at any instant $k$ are given by

$\underline{A}^{k}=\sum_{j=1}^{N} \lambda_{j}^{k} \varphi_{j}\left(A^{0}\right) \underline{V}_{j}$

Note that no model identification nor time integration is required to determine the chronos. Moreover, it is possible to reduce the number of terms involved in the summation by proposing a truncation of the CK modes based on an energy criterion. This truncation can be operated thresholding the energy spectrum to properly separate from the analysis the high frequency modes suspected to be related to 

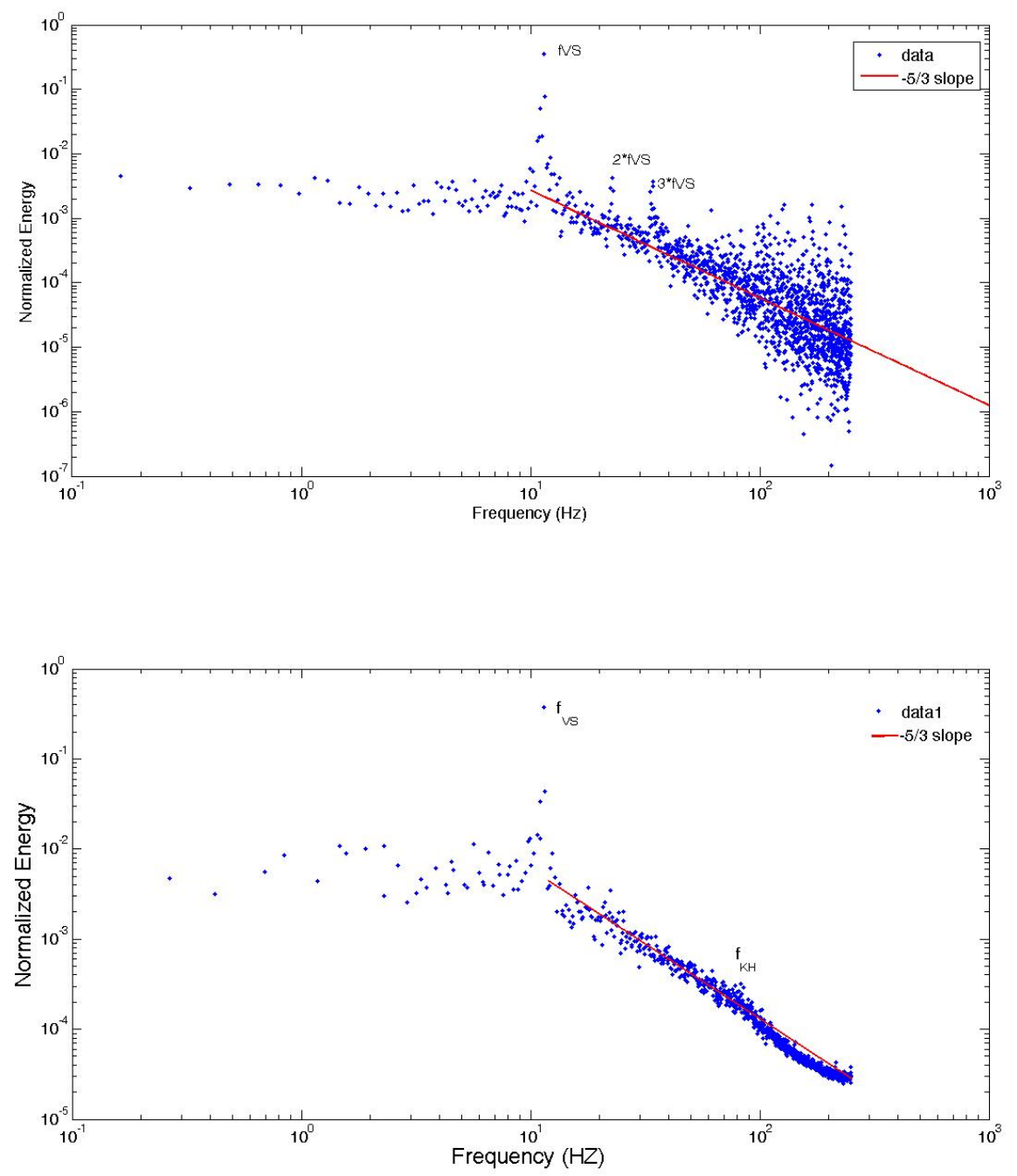

Fig. 11 Normalized Energy spectra of CK modes. a)Case I, b) Case II.

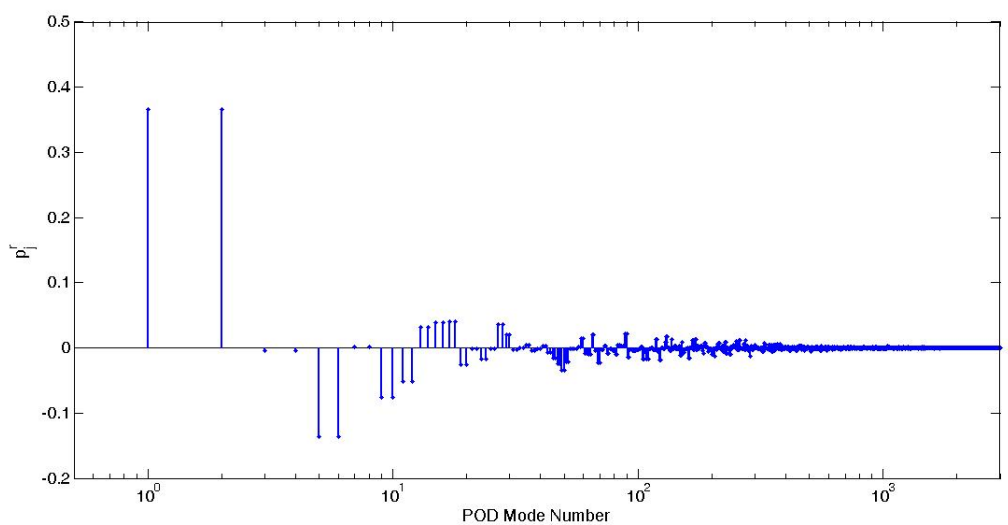

Fig. 12 Real Part of CK modes with associated frequency $f_{V S}=11.39 \mathrm{~Hz}$. 


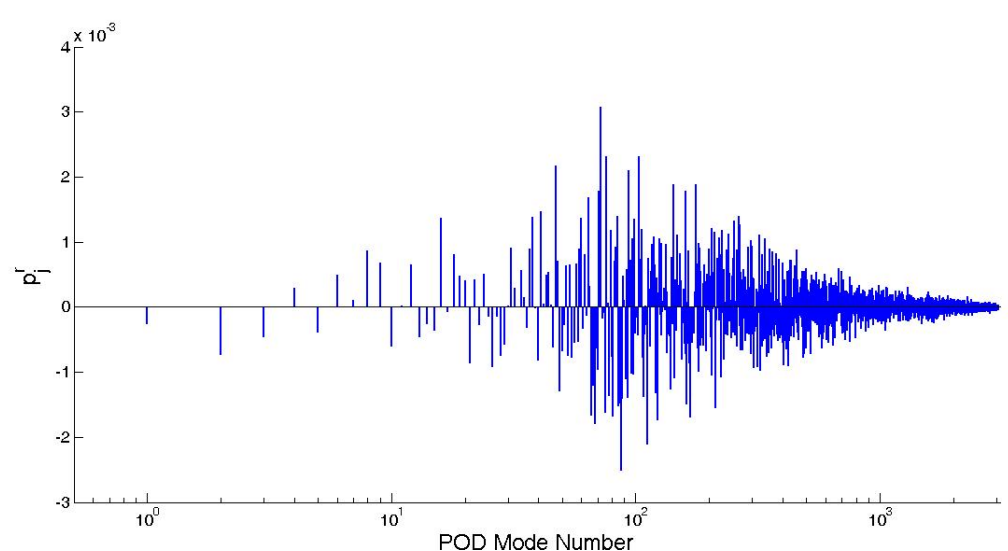

Fig. 13 Real Part of PK modes with associated frequency $f_{K H}=82.99 H z$.

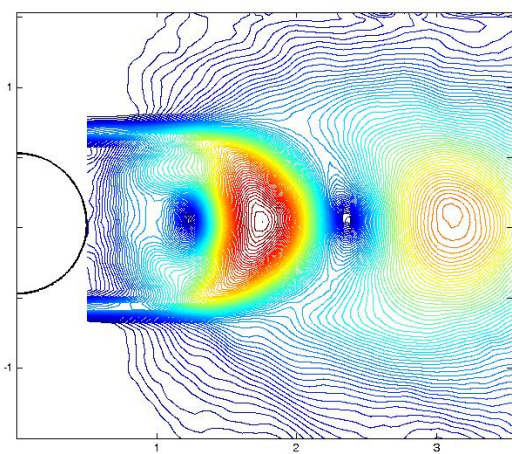

Fig. 14 Spatial structure associated with CK modes at $f_{V S}=11.39 \mathrm{~Hz}$. Isocontours of the norm of velocity field.

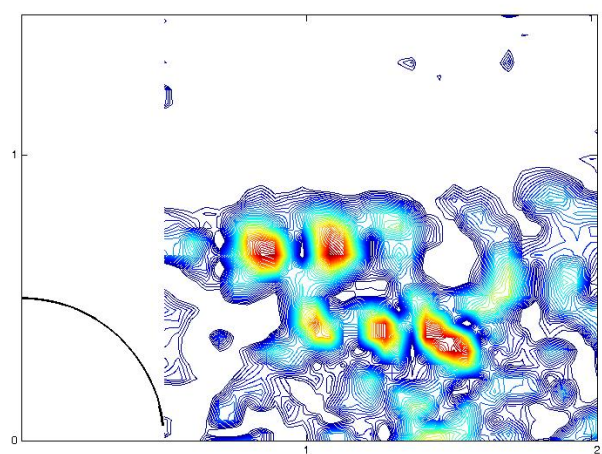

Fig. 15 Spatial structure associated with CK modes at $f_{K H}=82.99 H z$. Isocontours of the norm of velocity field.

noise.

\subsubsection{Stability of the model}

We can observe in Figure 16 and Figure 17 the time evolution of the POD modes and the values predicted using the reduced order model described by equation (36). We consider in figure 16 a 
system that includes all the CK modes (3070 CK modes), each one of these modes oscillating with its own frequency. On figure 17 we consider a truncated system composed by the modes having a normalized energy greater than 0.0001 (1230 CK modes were kept). On Figures 16a) and Figure 17a) we represent the predictions of the model at different times considering the whole data set. Both figures show a remarkable agreement between observations and values issued from the model. This agreement is also observed for modes of higher order of the POD. Note that the represented interval is very long (about 7 seconds) and that the period for which snapshots are available are more than 60 vortex shedding cycles (about 6 seconds). Problems of system stabilization that usually occur with POD-Galerkin methods are not encountered with our approach. The system is stable and does not diverge in the interval of observations without any tunning even in the truncated system.

At times much larger than those corresponding to observations, the system may exhibit a slight tendency to diverge. This effect is associated with an influence of the eigenvalues that are slightly larger than unit. However stability at very long times can be recovered by forcing these eigenvalues to lie on the unit circle.

Once we determine the time evolution of the temporal POD coefficients it becomes easy to reconstruct at any time the dynamics of velocity field or any derived quantity like for instance vorticity. Reconstruction is achieved for any instant superposing the topos weighted by the corresponding values of the chronos. As an example we show an image of instantaneous vorticity (Figure 18a) for the system with $3070 \mathrm{CK}$ modes and for the truncated system with $1230 \mathrm{CK}$ modes (Figure 18b) that can be compared to original data of Figure 2. The reconstruction is quite remarkable specially considering that the comparison is done with a derived quantity and that reconstruction involves the instantaneous values of a very large number of components of the observables. To illustrate the quality of reconstructions along the time, a film is also available with this document (Online Resource 1). This animation shows original vorticity issued from observations (upper frame) and the vorticity predicted using the full model (bottom left frame) and truncated model (bottom right frame). For model predictions regularly spaced intermediate reconstructions, at a number of three between observation delays, are included. As expected from figure 16 and figure 17 very good predictions of the vorticity in the flow is attained by the model and the reconstructions are very satisfactory at any instant in both cases.

\subsubsection{Model prediction at intermediate states of the learning period}

To check the adequacy of the model to predict intermediate states of the learning period we performed a second CK analysis, with a half and a third of snapshots of the sequence, thus artificially reducing the sampling rate. It is then possible to confront the predictions against the measured values. The results can be observed on figure $16 \mathrm{~b}$ ) and $17 \mathrm{~b}$ ). The agreement is remarkable with no significant differences even for the truncated system.

\subsubsection{Short term tracking}

Reduced order models are tools ideally suited for different applications like gaining insight into temporal dynamics and the motion of coherent structures, for understanding the energy exchange mechanisms between small and large scales and for isolating the physical mechanisms involved in transition.

The study of short time tracking of a model is of interest when it is desired to control the dynamics of coherent structures with actuators. For systems in which there may be a significant time lag between identification of the state of the system and the control action it becomes necessary to predict the state of the system at the future time when the control action will be effected. The model then must be capable of short term predictions.

Being the flow considered turbulent and with noisy initial conditions it is expected that there will always exist a rate of separation of trajectories in the phase space between the real dynamical system and predictions of the reduced model. It is of course futile for any system with a dynamic determined by a strange attractor, to try to track the state of coherent structures of the full fluid flow in the long term. This dynamical feature imposes to regularly recalibrate the initial state, before continuing prediction. 

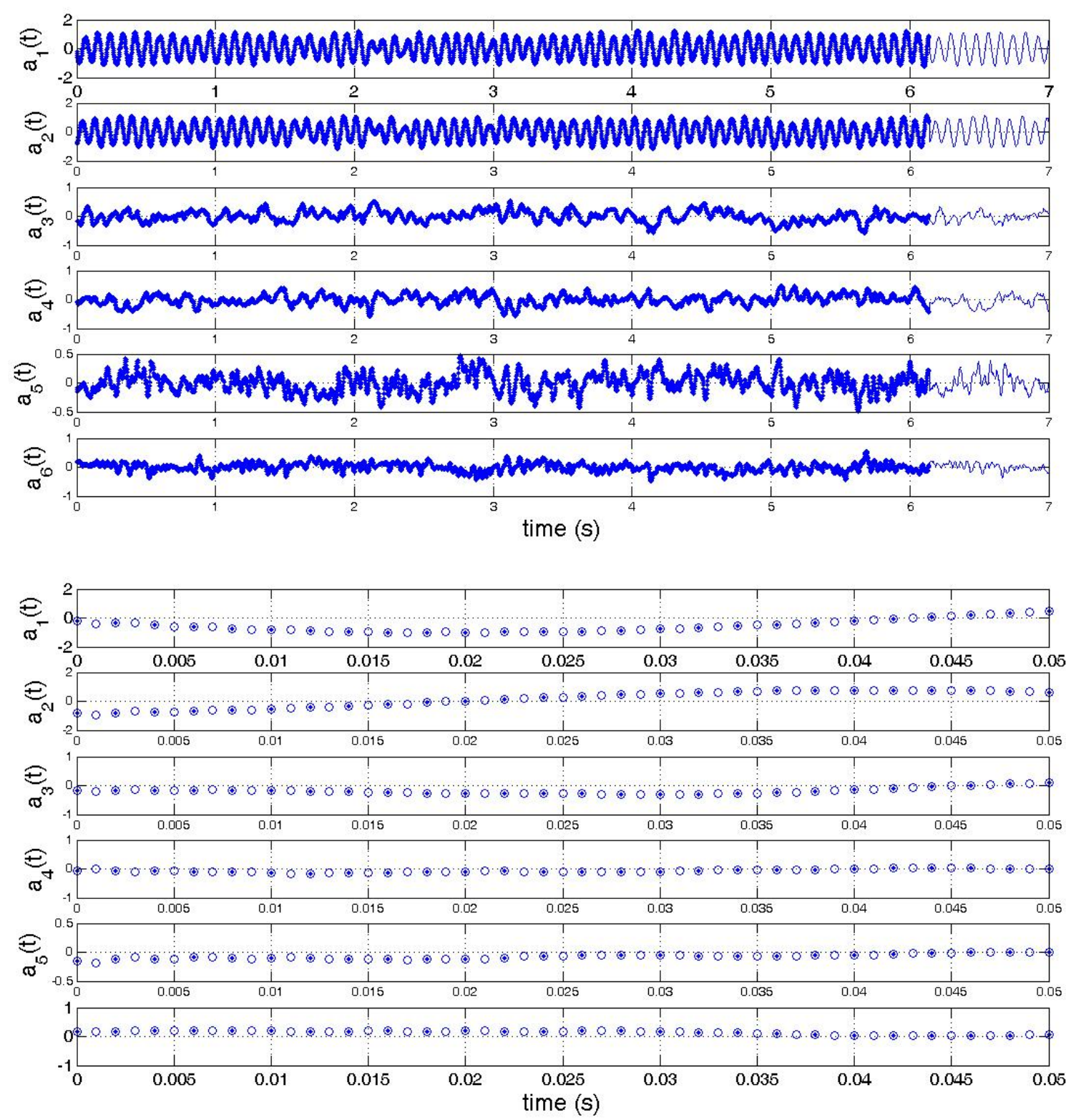

Fig. 16 Time evolution of temporal modes of POD and predictions by Chronos-Koopman spectral analysis (3070 CK modes).a) for long time period, b) Time zoom, filled markers are observations, hollow markers are predictions by the model

For the short term and for a given realisation of the fluid velocity field taken as initial condition, it is expected to obtain predictions of a low dimensional model that enable the tracking during a lapse of time.

The error represented by the difference between real and modeled flows grows with time and it is of interest to consider an average tracking error under a certain norm. In short time tracking at times $t$ larger than the learning period, the error can be evaluated by the following phase space norm.

$\zeta\left(\underline{u}_{0}, t_{n}\right)=\frac{\left\|A\left(t_{n}\right)-P \underline{u}\left(t_{n}\right)\right\|}{\left\|P \underline{u}\left(t_{n}\right)\right\|}$

where $A\left(t_{n}\right)$ is associated with the predictions of the model and $P$ accounts for the projection of instantaneous velocity field $\underline{u}\left(t_{n}\right)$ onto the POD modes calculated in the learning period. This error 

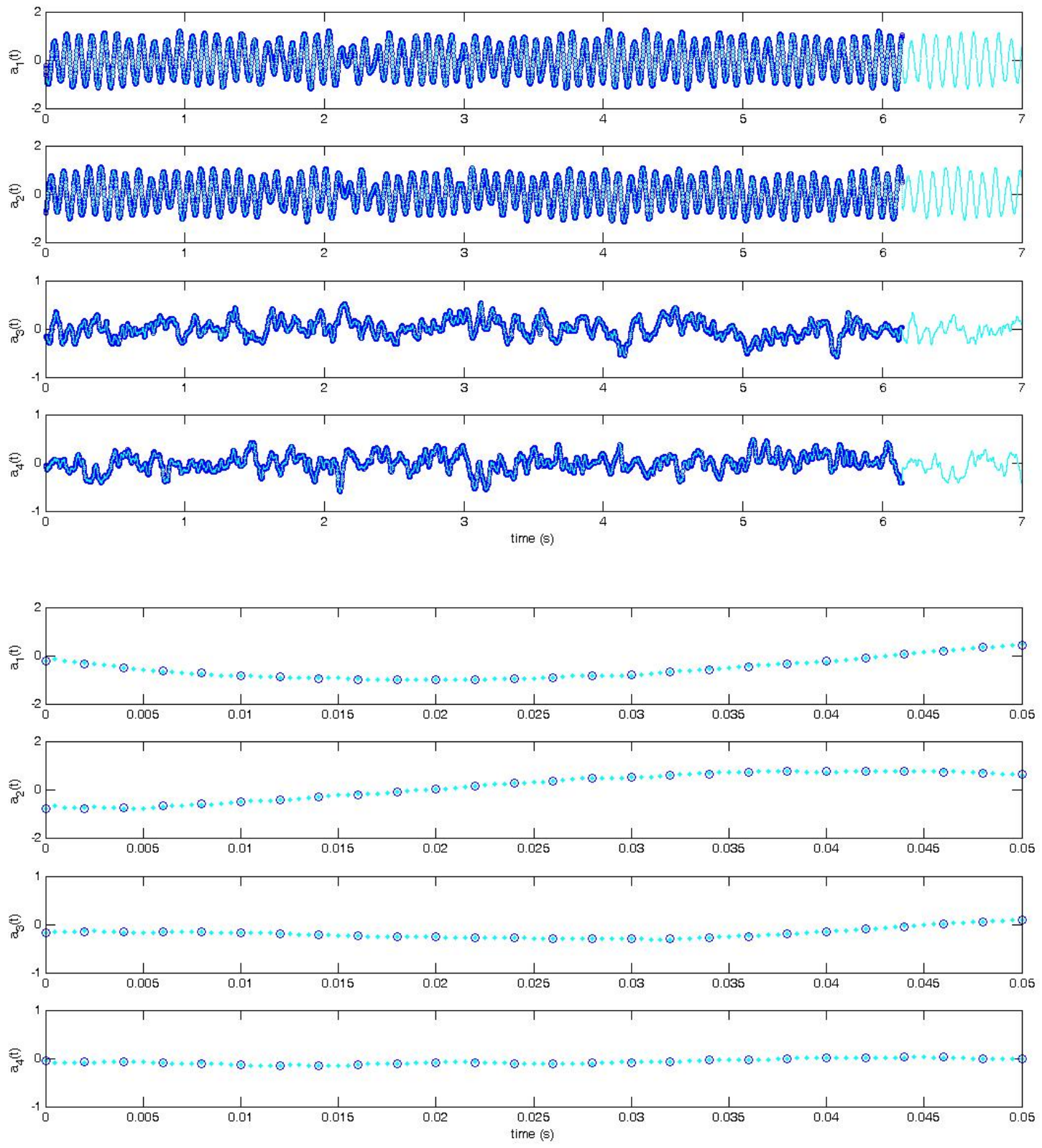

Fig. 17 Time evolution of temporal modes of POD and predictions by Chronos-Koopman spectral analysis with a truncated model (1230 CK modes).a) for long time period, b) Time zoom, filled markers are observations, hollow markers are predictions by the model

can be bounded in general by the following expression [19]

$\zeta\left(\underline{u}_{0}, t_{n}\right)<(K / L) \exp \left(L\left(t_{n}-t_{0}\right)\right)$

where $K$ and $L$ are constants.

The error depends on the considered initial conditions $\underline{u}_{0}$. It is convenient to undertake an average over all initial conditions likely to occur. We calculated the error for a set of initial conditions that lie in a vortex shedding period and averaged them. Taking as initial conditions all those corresponding to a vortex shedding cycle starting at time $=5 \mathrm{~s}$ and fitting the error with a least square technique gave us the following averaged values $\mathrm{K}=8.02 \mathrm{~s}^{-} 1$ and $\mathrm{L}=11.3 \mathrm{~s}^{-} 1$. Other tests undertaken with initial conditions at larger times or with the truncated model gave similar results. 

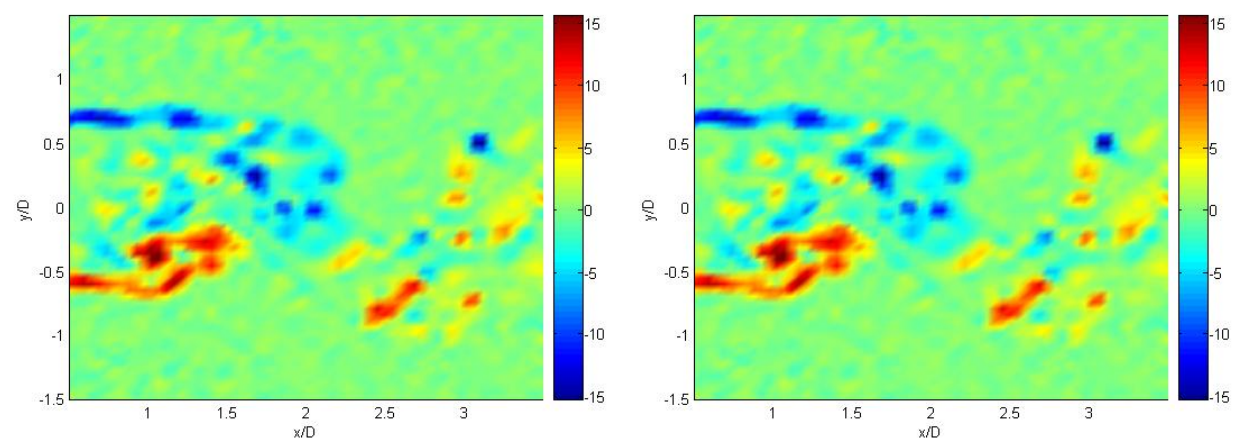

Fig. 18 Vorticity field reconstruction for the same snapshot of Figure 2. a)with 3072 CK modes b) with 1230 CK modes

These results indicate that differences may become significant in our case only when the elapsed time exceeds about half a cycle. For recalibration purposes, this value can be taken as a good indicator of the upper limit of time in which it is expected to have an accurate tracking. Note however, as can be observed in Figures 19, that the short term model expressed by (36) could still be capable to reproduce relatively well the dynamics of the most energetic structures of the flow even for times larger than half a cycle.
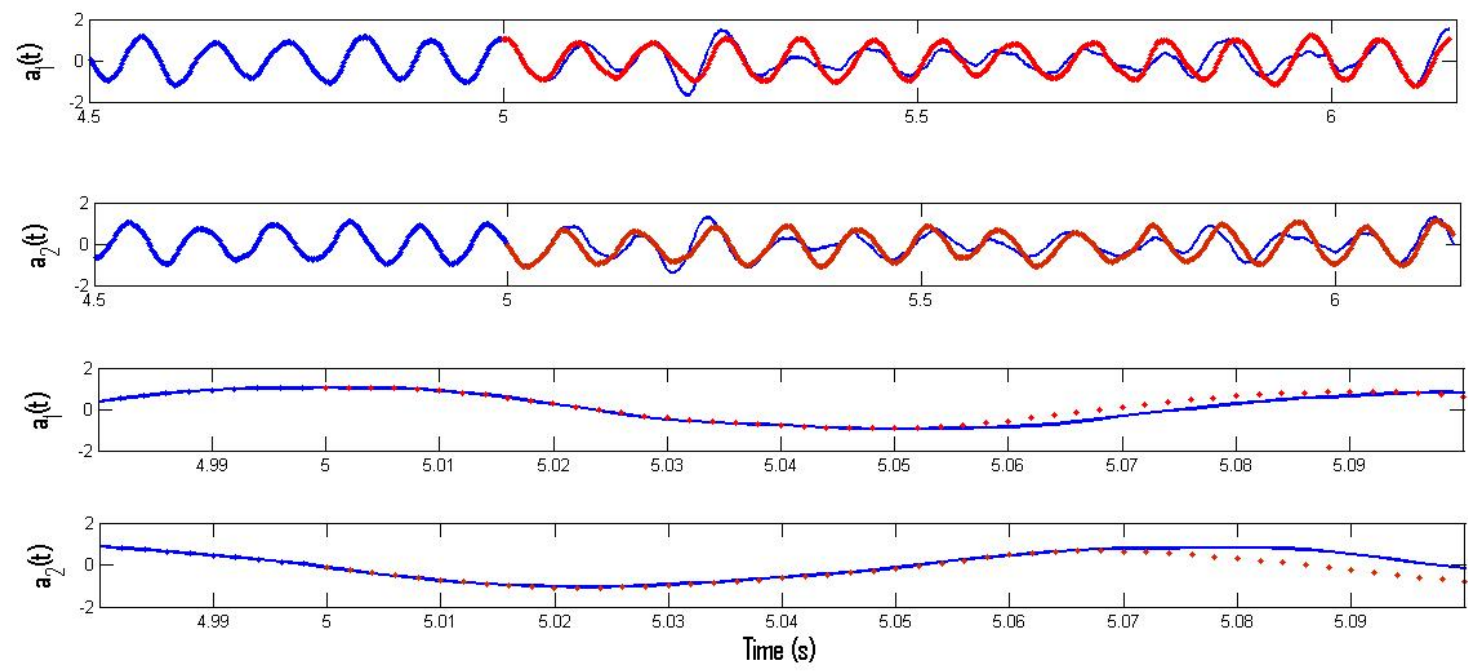

Fig. 19 Time evolution of temporal modes of POD and predictions by Chronos-Koopman spectral analysis with a learning period of $5 \mathrm{~s}$ (2500 CK modes).a) for long time period, b) Time zoom; filled markers are observations, continuous lines are predictions by the model

\section{Conclusions}

In this work we analyse the possibility to obtain stable reduced order modeling of flows using time data series of velocity fields. For this purpose we propose an algorithm that combines Proper Orthogonal Decomposition with spectral methods applied on the POD time-coefficients. On one hand, this algorithm allows to keep one of the main advantages of the POD: the energy computation of the modes. On the other hand, it overcomes the lack of bijective relationship between single frequencies 
and POD modes. Thus in order to decide which modes are relevant to describe the dynamics of the flow, a selection can be proposed based on the energy spectra. An inspection of the associated spatial modes helps to identify the flow features that can be associated with the frequency of interest.

Compared to traditional DMD in which the considered observables are snapshots of the velocity fields, our technique is particular of interest when the number of snapshots is much lower than the dimension of the snapshot vector or when eigenvalues do not lie into the unit circle in the complex plane.

We have illustrated our approach considering time resolved PIV data of a cylinder wake at $\operatorname{Re}=3900$. Looking at the kinetic energy spectrum, the peaks of which are well separated, it is easy to clearly observe the inertial subrange that extends over about a decade. The two predominant non stationary features of the flow, corresponding respectively to the vortex shedding and the shear instability frequencies can be identified. A classical DMD analysis undertaken directly over the velocity field components, did not enable to recognize the inertial subrange and frequencies of modes associated with shear layer instabilities.

Assuming that the emergence of a same given frequency in some POD modes expresses their effective coupling, we find which of the POD modes are coupled to the most distinctive instabilities of the flow. Vortex shedding instability involved a more reduced number of POD modes than shear layer instability mode.

A suitable model reduction could be obtained with a truncated system that excluded about $2 / 3$ of the total number of CK modes. No tunning was required to obtain this model stabilization. In the case studied, a precise tracking of the most energetic structures of the flow was possible when finished the learning period in a time horizon of about half a cycle.

Reconstruction of magnitudes derived from the velocity field, as vorticity, were also satisfactorily reproduced at intermediate states for which it was available the data of the system.

In conclusion, we think that this kind of analysis opens the possibility to post-process and analyze experimental and numerical data even in complex problems. The extension of the model reduction here proposed to flow control problems is matter of future studies.

\section{References}

1. A.Deane, I.Kevrekidis, G.Karniadakis, S.Orszag: Low-dimensional models for complex geometry fows: Application to grooved channels and circular cylinders. Physics of Fluids 3(10), 2337 (1991)

2. A.Prasad, C.Williamson:: The instability of the shear layer separating from a bluff body. Journal of Fluid Mechanics 333, 375-402 (1997)

3. B.Koopman: Hamiltonian systems and transformations in Hilbert space. Proc. Nat. Acad. Sci. USA 17, 315-318 (1931)

4. B.Noack, K.Afanasiev, M.Morzynski, G.Tadmor, F.Thiele: A hierarchy of low-dimensional models for the transient and post-transient cylinder wake. Journal of Fluid Mechanics 497, 335-363 (2003)

5. C.Rowley, I.Mezic, S.Bagheri, P.Schlatter, D.Henningson:: Spectral analysis of nonlinear flows. Journal of Fluid Mechanics 641, 115-127 (2009)

6. C.Rowley, I.Mezic, S.Bagheri, P.Schlatter, D.Henningson: Seventh IUTAM Symposium on LaminarTurbulent Transition, Vol. 18: Reduced-order models for flow control: balanced modes and Koopman modes. IUTAM Bookseries (2010)

7. F.Fang, C.C.Pain, I.M.Navon, M.D.Piggott, G.J.Gorman, A.J.H.Goddard:: Reduced order modelling of an adaptive mesh ocean model. Int. J. Num. Meth. in Fluids 59(8), 827-851 (2009)

8. F.Takens: Lecture Notes in Mathematics:Detecting Strange Attractors in Turbulence, Symp. Dynamical Systems and Turbulence. Springer-Verlag (1981)

9. G.Artana, A.Cammilleri, J.Carlier, E.Memin: Strong and weak constraint variational assimilations for reduced order fluid flow modeling. Journ of Comp Phys 9, To appear in (2011)

10. G.Berkooz, P.Holmes, J.Lumley: The proper orthogonal decompositon in the analysis of turbulent flows. Annu. Rev. Fluid. Mech 25, 539-575 (1993)

11. G.Dergham, D., J.C.Robinet, A.Barbagallo: Model reduction for fluids using frequential snapshots. Physics of Fluids 23(6), 64,101 (2011)

12. G.Karamanos, G.Karniadakis: A spectral vanishing viscosity method for large-eddy simulations. Journal of Computational Physics 163(1), 22-50 (2000)

13. H.Aling, S.Banerjee, A.K.Bangia, V.Cole, J.L.Ebert, A.Emami-Naeini, K.F.Jensen, I.G.Kevrekidis, S.Shvartsman: Nonlinear model reduction for simulation and control of rapid thermal processing. Proceedings of the American Control Conference 8, 2233-2238 (1997)

14. I.Mezic: Spectral properties of dynamical systems, model reduction and decompositions. Nonlinear Dynamics 41(1-3), 309-325 (2005) 
15. J.D'Adamo, N.Papadakis, E.Memin, G.Artana: Variational assimilation of pod low-order dynamical systems. Journal of Turbulence $\mathbf{8 ( 9 )}, 1-22(2007)$

16. J.Ding: The point spectrum of frobenius-perron and kooman operators. Proc Amer Math Soc 126(5), 1355-1361 (1998)

17. K.K.Chen, Tu, J.H., Rowley, C.W.: Variants of dynamics modes decomposition boundary condition, koopman and fourier analysis. J. Nonlinear Sci. 1, 1-2 (2012)

18. M.Bergmann, L.Cordier: Optimal control of the cylinder wake in the laminar regime by trust-region methods and pod reduced-order models. Journ. of Comp. Physics 227(16), 7813-7840 (2008)

19. P.Holmes, J.L.Lumley, G.Berkooz: Turbulence, coherent structures, dynamical systems and symetry. Cambridge University Press (1996)

20. P.Moin, R.Moser: Characteristic eddy decomposition of turbulence in a channel. J. Fluid Mech. 200, 471-509 (1989)

21. P.Parnaudeau, J.Carlier, D.Heitz, E.Lamballais: Experimental and numerical studies of the flow over a circular cylinder at Reynolds number 3 900. Physics of Fluids 20(8), 085,101 (2008)

22. P.Schmid, L.Li, M.Juniper, O.Pust: Applications of the dynamic mode decomposition. Theoretical and Computational Fluid Dynamics p. (in press) (2011)

23. S.Bagheri: Analysis and control of transitional shear flows using global modes. Phd Thesis, Royal Institute of Technology, Stocholm, Sweden (2010)

24. Schmid, P.: Dynamic mode decomposition of numerical and experimental data. Journal of Fluid Mechanics 656, 5-28 (2010)

25. S.Dong, G.Karniadakis, A.Ekmekci, D.Rockwell: A combined direct numerical simulation-particle image velocimetry study of the turbulent near wake,. J. Fluid Mech. 569, 185-207 (2006)

26. Sirovich, L.: Turbulence and the dynamics of coherent structures. Quarterly of Applied Mathematics XLV, $561-590(1987)$

27. V.Kalb, A.Deane:: An intrinsic stabilization scheme for proper orthogonal decomposition based lowdimensional models. Physics of fluids 19(5), 54,106 (2007)

28. X.Ma, G.Karamanos, G.Karniadakis: Dynamics and low-dimensionality of a turbulent near wake. J. Fluid Mech. 410, 29-65 (2000)

29. Y.Cao, J.Zhu, I.M.Navon, Z.Luo: A reduced order approach to four-dimensional variational data assimilation using proper orthogonal decomposition. Int. J. Num. Meth. in Fluids 53(10), 1571-1583 (2007)

30. Zdravkovich:, M.: Flow around circular cylinders: Vol 1 Fundamentals. Oxford Science Publication Oxford (1997) 